\title{
Predicting the distribution of canine leishmaniasis in western Europe based on environmental variables
}

\author{
ANA O. FRANCO ${ }^{1} \dagger$, CLIVER. DAVIES ${ }^{1} \dagger \dagger$, ADRIAN MYLNE ${ }^{1}$, JEAN-PIERRE DEDET $^{2}$, \\ MONTSERRAT GÁLLEGO ${ }^{3}$, CRISTINA BALLART ${ }^{3}$, MARINA GRAMICCIA $^{4}$, \\ LUIGI GRADONI $^{4}$, RICARDO MOLINA ${ }^{5}$, ROSA GÁLVEZ ${ }^{5}$, \\ FRANCISCO MORILLAS-MÁRQUEZ ${ }^{6}$, SERGIO BARÓN-LÓPEZ ${ }^{6}$, CARLOS \\ ALVES PIRES ${ }^{7}$, MARIA ODETE AFONSO $^{7}, \mathrm{PAUL}^{\mathrm{D}}$. READY ${ }^{*}$ and JONATHAN COX ${ }^{1} \dagger$ \\ ${ }^{1}$ Faculty of Infectious and Tropical Diseases, London School of Hygiene and Tropical Medicine, London, UK \\ ${ }^{2}$ Centre National de Référence des Leishmania, UMR MIVEGEC, Université Montpellier 1/Laboratoire de \\ Parasitologie-Mycologie, CHU de Montpellier, Montpellier, France \\ ${ }^{3}$ Laboratori de Parasitologia, Facultat de Farmàcia, Universitat de Barcelona, Barcelona, Spain \\ ${ }_{5}^{4}$ Unit of Vector-borne Diseases and International Health, MIPI Department, Istituto Superiore di Sanità, Rome, Italy \\ ${ }^{5}$ Laboratorio de Referenda de Leishmaniasis, Servicio de Parasitología, Centro Nacional de Microbiología, Instituto de Salud \\ Carlos III, Madrid, Spain \\ ${ }^{6}$ Departamento de Parasitología, Facultad de Farmacia, Universidad de Granada, Granada, Spain \\ ${ }^{7}$ Unidade de Entomologia Médica/Unidade de Parasitologia e Microbiologia Médica, Instituto de Higiene e Medicina \\ Tropical, Universidade Nova de Lisboa, Lisbon, Portugal \\ ${ }^{8}$ Department of Entomology, Natural History Museum, London, UK
}

(Received 10 Fune 2011; accepted 1 August 2011; first published online 14 September 2011)

SUMMARY

The domestic dog is the reservoir host of Leishmania infantum, the causative agent of zoonotic visceral leishmaniasis endemic in Mediterranean Europe. Targeted control requires predictive risk maps of canine leishmaniasis (CanL), which are now explored. We databased 2187 published and unpublished surveys of CanL in southern Europe. A total of 947 western surveys met inclusion criteria for analysis, including serological identification of infection (504, 369 dogs tested 1971-2006). Seroprevalence was $23 \cdot 2 \%$ overall (median 10\%). Logistic regression models within a GIS framework identified the main environmental predictors of CanL seroprevalence in Portugal, Spain, France and Italy, or in France alone. A 10-fold cross-validation approach determined model capacity to predict point-values of seroprevalence and the correct seroprevalence class $(<5 \%, 5-20 \%,>20 \%)$. Both the four-country and France-only models performed reasonably well for predicting correctly the $<5 \%$ and $>20 \%$ seroprevalence classes (AUC $>0 \cdot 70$ ). However, the France-only model performed much better for France than the four-country model. The four-country model adequately predicted regions of CanL emergence in northern Italy $(<5 \%$ seroprevalence). Both models poorly predicted intermediate point seroprevalences (5-20\%) within regional foci, because surveys were biased towards known rural foci and Mediterranean bioclimates. Our recommendations for standardizing surveys would permit higher-resolution risk mapping.

Key words: canine leishmaniasis, western Mediterranean, predicting distribution, serological records, seroprevalence.

\section{INTRODUCTION}

Zoonotic visceral leishmaniasis (ZVL) is endemic in Mediterranean Europe, where the causative agent Leishmania infantum is transmitted by phlebotomine sandflies of the subgenus Phlebotomus (Larroussius) (Ready, 2010), but its surveillance and control are neglected compared with research efforts (Dujardin et al. 2008). There are estimated to be only 700 new

\footnotetext{
* Corresponding author: Department of Entomology, Natural History Museum, London SW7 5BD, UK. Tel: + 442079425622. Fax: + 442079425229. E-mail: P.Ready@nhm.ac.uk

+ Except for these two, the authors are listed in alphabetical order of team leaders, each of whom is followed by any team member involved in databasing. †† Clive Davies passed away in March 2009.
}

human cases of ZVL per year in southern Europe (Dujardin et al. 2008), and some people develop cutaneous lesions. However, the seroprevalence in the domestic dog, the only proven reservoir host (Quinnell and Courtenay, 2009), is often 20\% (Dujardin et al. 2008), sufficiently high to pose a serious risk of re-emergence of human disease, especially in immuno-suppressed people (Ready, 2010). Human cases are not usually infectious to sandfly vectors, but the parasite has been exported from Europe in the canine reservoir hosts, historically to Latin America where it now causes much fatal infantile disease (Romero and Boelaert, 2010) and recently even to North America in fox hounds (Duprey et al. 2006).

The control of canine leishmaniasis (CanL) is considered to be the best way of reducing the 
incidence of human disease in ZVL foci (Quinnell and Courtenay, 2009) and one way of controlling any spread northwards in Europe (Ready, 2010). Targeted control would be assisted by predictive risk maps of CanL, but unfortunately these do not exist, even in Europe where there have been numerous surveys and incidence has been mapped for positive point locations or administrative areas (Trotz-Williams and Trees, 2003). The current report investigates the challenges of producing a predictive risk map of CanL for western Europe, based on the authors' databasing of historical records of CanL during the EU EDEN project (Emerging Diseases in a changing European eNvironment; http://www. eden-fp6project.net). Records for Eastern Europe were included in our database, but they are not analysed in the current report because they are relatively few in number and the transmission cycles are biogeographically distinct (Ready, 2010). Only 2 sandflies, Phlebotomus perniciosus and P. ariasi, have been incriminated as vectors in Portugal, Spain and France, and the former is also the most widespread vector in Italy, where $P$. perfiliewi and P. neglectus pose regional threats. Further east, the vectors are $P$. perfiliewi, $P$. neglectus and P. tobbi.

Of all the CanL records we found in the literature and personal files, only those from random surveys of $\mathrm{dog}$ populations were targeted for our risk mapping, in order to lessen the chances of analysing populations of mixed geographical origins, including travel cases, or biasing prevalence estimates by missing asymptomatic seropositive dogs. Multivariate logistic regression models were built, using a GIS framework, to find the main predictors of CanL prevalence in western Europe or France alone based on a range of variables, including remotely-sensed climatological and vegetation indices.

\section{MATERIALS AND METHODS}

\section{Developing a European database of CanL}

The EDEN subproject on leishmaniasis developed a database of all surveys of CanL carried out in Europe and other Mediterranean countries from 1965. A standardized Microsoft ${ }^{\circledR}$ Office Excel worksheet included information related to: publication or dataset; survey type, date, location and environment; method of selecting dogs to be screened; method(s) of diagnosis of infection; dog travel history, breed, age and lifestyle; number of dogs tested and number positive.

For the present study, the data-gathering teams in Portugal (1), Spain (3), France (1) and Italy (1) performed a systematic search for published and unpublished reports in their regions, based on their expert knowledge of data sources. Data entries were standardized by the analytical team in the UK, based on clarifications provided by the data gatherers. The present analysis included only surveys that provided estimates of CanL prevalence based on serological diagnosis. Surveys were excluded from the analysis if 1 or more of the following criteria applied: CanL prevalence could not be estimated either directly or indirectly; the method of diagnosis was not serological (but was based solely on clinical signs, microscopy or molecular characterization); dogs were clearly not sampled 'randomly' from a settlement or administrative unit (excluded were positive cases reported by veterinary clinics or other cases possibly associated with passive detection and unknown combinations of localities); data were duplicated and reported more completely in another publication; location was missing or unable to be geo-positioned; and, environmental data were lacking.

Only a minority of reports included geographical coordinates. Most records mentioned the nearest settlement, which was geo-positioned using Google Earth (http://earth.google.com; accessed 2 February 2009). The survey date was potentially an explanatory variable, and so it was derived by (a) averaging the survey's start and end years, or (b) using one of those two dates if the other was missing, or (c) using the year of publication if survey dates were not reported.

\section{Environmental data}

Data for long-term climatic averages (based on the period 1961-1990) of monthly precipitation, relative humidity, mean temperature and mean diurnal temperature range were obtained at a spatial resolution of 10 arc-minutes from the Climatic Research Unit, University of East Anglia, UK (New et al. 2002). Monthly data were aggregated into averages for the warm months (May to September) and for the cold months (October to April). Remotely sensed (MODIS v4), Fourier-processed data (Rogers et al. 1996) for day-time (LSTD) and night-time (LSTN) land surface temperature, middle infrared reflectance (MIR) and Enhanced vegetation index (EVI) for the period 2001-2005 were produced by Professor David Rogers and his team (Spatial Ecology and Epidemiology Research Group, Department of Zoology, Oxford University) and obtained from the EDEN project data archive (http://ergodd.zoo.ox.ac. uk/EDEN/index.php?p=1; accessed 2 March 2009). These data have a spatial resolution of $1 \mathrm{~km}(250 \mathrm{~m}$ for EVI) and include separate products relating to the means, amplitudes and phases of annual, biannual and tri-annual cycles. Elevation data were derived from the GLOBE digital elevation model (spatial resolution $1 \mathrm{~km}$ ) (US National Geophysical Data Center (NGDC), National Oceanic \& Atmospheric Administration (NOAA) Satellite and Information Service. Available: http://www.ngdc.noaa.gov/; accessed 2 March 2009). 


\section{Statistical model building}

CanL seroprevalence was modelled as a binomial variate: the number of dogs examined and dogs found exposed to Leishmania infection at each survey location. To overcome the dependency of observations at the same location, the association between risk of exposure to infection and the environmental covariates was determined using fixed-effects grouped logistic regression with robust standard errors (Rogers, 1993; Rabe-Hesketh and Everitt, 2004) in the statistical package Stata (version 10; StataCorp, College Station, Texas, USA). Binary logistic regression (logit command in Stata) is more frequently used for statistical modelling, but this would have required splitting CanL seroprevalence into 2 categories and, thereby, losing information (Rabe-Hesketh and Everitt, 2004). In addition, global spatial structures within the seroprevalence data and among model Pearson residuals from multivariate regression analysis were evaluated using semivariograms estimated using the $\mathrm{R}$ module GeoR (Ribeiro and Diggle, 2001). Semivariograms show the spatial dependence of the variable of interest as a scatter plot, and as such provide a means of assessing visually the presence of spatial autocorrelation (Pullan et al. 2008).

Prior to multivariate analysis, univariate binomial logistic regression analyses were carried out to test for associations between seroprevalence and each of the 46 environmental variables, as well as the potentially confounding variable of the survey country. Predictors that were significant at the $10 \%$ probability level were retained. The uncentred variance inflation factor (VIF) was used to screen for co-linearity between the retained environmental continuous variables (Rabe-Hesketh and Everitt, 2004). Within a group of themed environmental variables, those with VIF $\geqslant 10$ were excluded one by one. The procedure was repeated to test for co-linearity between the variables of different groups. The remaining variables were included in a multivariate binomial logistic regression model, for which a backward stepwise selection procedure was performed, excluding the variables with the highest Wald test $P$-value until all the variables in the model had $P<0 \cdot 05$.

Some of the variables retained in the model were non-linear, and these were transformed if this improved the model fit. We found the best transformation for each predictor, starting with the one with the smallest $P$-value in the linear model, and then transforming the predictor with the next smallest $P$-value. The non-linearity of each variable (x) was explored by a quadratic polynomial transformation and by 7 fractional polynomial transformations of the first degree (FPs), namely $1 / \mathrm{x}^{2}, 1 / \mathrm{x}, 1 / \mathrm{x}^{0 \cdot 5}$, $\ln (\mathrm{x}), \mathrm{x}^{0 \cdot 5}, \mathrm{x}^{2}$, and $\mathrm{x}^{3}$ (Royston et al. 1999; Hosmer and Lemshow, 2000). The best-fit transformation is the one that produces the largest reduction in the residual deviance. The quadratic polynomial transformation was selected if it decreased deviance by more than 5.99 compared with the best fit FP. Otherwise, the best fit FP was selected if it decreased deviance by more than 3.84 compared with the linear (i.e. untransformed) variable. If the decrease in deviance was less, then the variable remained linear. This process was repeated several times until no further FP transformations were indicated for the multivariate model without clustering, and then the same transformation was applied when fitting the final model with clustering (Hosmer and Lemshow, 2000).

The same method was used to build 2 additional models. For the four-country dataset, a separate model included diagnostic method and survey year, to assess whether they improved the accuracy of the model predictions. A separate model was also built for the data from France alone, in order to investigate better any effect of country on accuracy.

\section{Model validation}

The predictive performance of each model was assessed by using a 10-fold cross-validation approach (Hastie et al. 2001; Tibshirani et al. 2002). Briefly, the CanL seroprevalence dataset was split randomly into 10 approximately equal-sized parts. The final multivariate logistic model was fitted on $90 \%$ of the data points, and then used to predict the seroprevalence of the remaining $10 \%$ (the validation set). This procedure was performed 10 times, each time with 1 of the 10 dataset parts acting as the validation set. The seroprevalences predicted by all the validation sets were then compared with the observed values for the same locations. For each of the validation sets the predictive performance of the model was tested by determining the capacity of the model to predict either point-values of seroprevalence - using the correlation coefficient as a measure of linear association between the predicted and the observed values (Gething et al. 2008; Hay et al. 2009) - or the correct endemicity class. Three endemicity classes were considered: low ( $<5 \%$ seroprevalence), medium $(5-20 \%)$, and high risk $(>20 \%)$. The capacity of the model to predict the correct endemicity class was assessed using the area-under-curve (AUC) of a receiver-operating-characteristic (ROC) curve, which plots sensitivity versus one minus specificity for each endemicity class (Brooker et al. 2002; Hay et al. 2009). AUC values indicate the agreement between the observed and predicted endemicity class: poor, 0.51-0.70; reasonable, 0.71-0.90; and excellent, $>0 \cdot 9$ (Brooker et al. 2002; Hay et al. 2009). The prediction accuracy was further assessed by a contingency table and by calculating the percentage of points classified in the correct endemicity class or 
in a non-adjacent class (Hay et al. 2009). The validation statistics for all 10 dataset parts were averaged to compute the overall accuracy of the predictions.

\section{Producing a risk map of CanL seroprevalence}

Each statistical model was then used to estimate CanL seroprevalence for a country, based on the predictor values available from remotely sensed images for a $1 \mathrm{~km}$-square grid. Finally, the predicted CanL risk was converted into 1 of 5 endemicity classes: low ( $<5 \%$ seroprevalence), medium-low (5-10\%), medium-high (10.01-20\%), high (20.01$30 \%)$ and very high risk $(>30 \%)$, and the predicted seroprevalence was mapped using ArcGIS v. 9.2 (ESRI, Redlands, CA, USA). Seroprevalence was mapped only for the pixels where the environmental values were within the range of those in the survey locations.

\section{RESULTS}

\section{Database of CanL in Europe}

The historical CanL database included crosssectional surveys, prospective surveys, laboratory records, cases reported at veterinary clinics and case reports. It contained 2187 surveys, including 33 from Croatia, 26 from Greece and 55 from Turkey. There was a total of 2073 reports from Portugal, Spain, France and Italy, of which 947 (45.7\%) were included and 1126 were excluded from the current analysis based on the criteria reported above. The most frequent exclusion reasons were non-random sampling $(n=849 ; 75 \cdot 4 \%)$, missing data for the number of dogs tested or positive $(n=595 ; 52 \cdot 8 \%)$ and non-serological diagnosis $(n=258 ; 22 \cdot 9 \%)$. Of the latter, 117 were solely based on microscopy, 58 on culture, 47 on molecular characterization, and 36 on clinical signs. The datasets with the included and excluded CanL seroprevalence surveys can be obtained from the corresponding author.

The remaining 947 surveys were analysed, and the reports included 124 publications. The surveys were undertaken between 1971 and 2006 inclusive (75\% after 1985 , and $50 \%$ after 1992 ) and involved 504369 dogs tested for exposure to Leishmania infections. The data analysed are summarized in Table 1 and mapped in Fig. 1. Of the analysed surveys, most were conducted in Italy $(377 ; 40 \%)$, followed by Spain $(213 ; 22 \%)$, Portugal $(188 ; 20 \%)$ and France (169; $18 \%)$. The median number of dogs tested per survey was 67, being highest in Italy (168) and lowest in Spain (39). The frequency distribution of the number of dogs per survey was highly right skewed, with the maxima being 30001 for Italy, 7067 for France, 1803 for Spain and 1024 for Portugal. Surveys were conducted between latitudes $35 \cdot 5^{\circ}$ and $47 \cdot 5^{\circ}$ North and longitudes $9 \cdot 3^{\circ}$ West and $17 \cdot 6^{\circ}$
East. The analysed records covered altitudes of $1-1838 \mathrm{~m}$ above sea level (median $204 \mathrm{~m}$ a.s.1.). Most surveys (99\%) were conducted below $1000 \mathrm{~m}$ a.s.1. (3 at $1000-1500 \mathrm{~m}$ a.s.1. in France; and 6 at 1000-1500 $\mathrm{m}$ a.s.1. and 1 at $1838 \mathrm{~m}$ a.s.l. in Spain). The diagnostic method most commonly used was the indirect fluorescent antibody test (IFAT), both overall $(77 \%)$ and in each country, and the most frequent IFAT cut-off was equal to or above the current minimum standard of 1:80. The proportion of surveys using an IFAT cut-off $<1: 80$ was $35 \cdot 4 \%$ in Italy, $19 \cdot 2 \%$ in Portugal, $2 \cdot 4 \%$ in Spain and $0 \%$ in France. The other serological diagnostic methods most frequently used were ELISA, DOT-ELISA and the direct antibody test (DAT).

The overall CanL seroprevalence was $23 \cdot 2 \%$ $(116,968 / 504,369)$, and $5 \%$ of the surveys recorded seroprevalences $>40 \%$. Point seroprevalences $>80 \%$ were recorded in Italy, Portugal and Spain, but the maximum in France was only $43 \%$. The median seroprevalence was $10 \%$, being highest in Italy $(17 \cdot 7 \%)$, followed by France $(8 \%)$, Portugal $(7 \cdot 3 \%)$ and Spain $(5 \cdot 9 \%)$. Zero seroprevalences were recorded in $14.7 \%$ of the surveys, being more frequent in Spain (25\%), followed by Portugal (20\%), France $(14 \%)$, and Italy $(6 \%)$.

\section{Statistical models}

Four-country model. Among the 49 variables screened by univariate analysis (Table 2), 32 were significantly associated with CanL seroprevalence at the $10 \%$ probability level: diagnostic method, survey year, country, altitude, latitude, 21 Fouriertransformed remotely sensed (RS) variables from 4 environmental groups, and 6 variables from 4 climatic groups. Of the latter 27 variables, 19 were excluded due to co-linearity, leaving 11 variables to be tested for inclusion in the multivariate model. These consisted of country, altitude, latitude and 8 Fourier-transformed RS variables relating to night-time land surface temperature (minimum (LSTNmn), amplitude of the tri-annual cycle (LSTNa3)), day-time land surface temperature (amplitude of the bi-annual cycle (LSTDa2), phase of the tri-annual cycle (LSTDp3)), and enhanced vegetation index (minimum (EVImn), amplitude of the annual cycle (EVIa1), and phases of the annual and tri-annual cycles (EVIp1, EVIp3)). Of these, 7 variables remained in the final multivariate model (Table 3). The association with seroprevalence was inversely $\mathrm{U}$-shaped for some predictors (i.e. the highest seroprevalence was observed at intermediate values), namely altitude, LSTNmn, LSTNa3 and to a much lesser degree EVIp1, and so all were used with a quadratic transformation. When testing for the confounding effect of diagnostic method and survey year, only the latter dropped from the multivariate 
Table 1. Seroprevalences of canine leishmaniasis and number of dogs tested for all surveys analysed

\begin{tabular}{|c|c|c|c|c|c|c|c|c|c|c|c|c|c|c|c|}
\hline \multirow{4}{*}{$\begin{array}{l}\text { No. surveys } \\
\text { Survey category } \\
\text { Total }\end{array}$} & \multicolumn{3}{|c|}{ All countries $(n=947)$} & \multicolumn{3}{|c|}{ France $(n=169)$} & \multicolumn{3}{|c|}{ Italy $(n=377)$} & \multicolumn{3}{|c|}{ Portugal $(\mathrm{n}=188)$} & \multicolumn{3}{|c|}{ Spain $(n=213)$} \\
\hline & \multicolumn{2}{|c|}{ Prevalence } & \multirow[t]{2}{*}{$\%$} & \multicolumn{2}{|c|}{ Prevalence } & \multirow[t]{2}{*}{$\%$} & \multicolumn{2}{|c|}{ Prevalence } & \multirow[t]{2}{*}{$\%$} & \multicolumn{2}{|c|}{ Prevalence } & \multirow[t]{2}{*}{$\%$} & \multicolumn{2}{|c|}{ Prevalence } & \multirow[t]{2}{*}{$\%$} \\
\hline & Median & (Range) & & Median & (Range) & & Median & (Range) & & Median & (Range) & & Median & (Range) & \\
\hline & $10 \cdot 0$ & $(0-100)$ & $100^{\mathrm{a}}$ & $8 \cdot 0$ & $(0-43 \cdot 3)$ & $17 \cdot 8^{\mathrm{a}}$ & $17 \cdot 7$ & $(0-100)$ & $39 \cdot 8^{\mathrm{a}}$ & $7 \cdot 3$ & $(0-81 \cdot 1)$ & $19 \cdot 9^{\mathrm{a}}$ & $5 \cdot 9$ & $(0-100)$ & $22 \cdot 5^{\mathrm{a}}$ \\
\hline \multicolumn{16}{|c|}{ Survey year } \\
\hline 1971-1980 & $6 \cdot 3$ & $(0-100)$ & $13 \cdot 8$ & $4 \cdot 4$ & $(0-40 \cdot 0)$ & $42 \cdot 6$ & $11 \cdot 1$ & $(0-100)$ & $12 \cdot 5$ & $0 \cdot 0$ & $(0-3 \cdot 6)$ & $6 \cdot 4$ & - & $(-)$ & - \\
\hline 1981-1990 & $10 \cdot 2$ & $(0-66 \cdot 7)$ & $28 \cdot 9$ & $13 \cdot 2$ & $(0 \cdot 7-43 \cdot 3)$ & $14 \cdot 8$ & $19 \cdot 1$ & $(2 \cdot 5-66 \cdot 7)$ & $9 \cdot 3$ & $10 \cdot 4$ & $(0-39 \cdot 8)$ & $57 \cdot 4$ & $6 \cdot 9$ & $(0-45 \cdot 5)$ & $49 \cdot 8$ \\
\hline 1991-2000 & $11 \cdot 7$ & $(0-100)$ & $45 \cdot 0$ & $9 \cdot 1$ & $(0-27 \cdot 6)$ & $34 \cdot 9$ & $21 \cdot 1$ & $(0-100)$ & $53 \cdot 6$ & $6 \cdot 3$ & $(0-81 \cdot 1)$ & $33 \cdot 0$ & $5 \cdot 2$ & $(0-100)$ & $48 \cdot 4$ \\
\hline \multirow{2}{*}{\multicolumn{16}{|c|}{ Altitude }} \\
\hline & & & & & & & & & & & & & & & \\
\hline $1-<100$ & $12 \cdot 5$ & $(0-100)$ & $32 \cdot 4$ & $7 \cdot 3$ & $(0-43 \cdot 3)$ & $28 \cdot 4$ & $16 \cdot 8$ & $(0-100)$ & $44 \cdot 8$ & $7 \cdot 1$ & $(0-34 \cdot 1)$ & $35 \cdot 6$ & $5 \cdot 9$ & $(0-34 \cdot 6)$ & $10 \cdot 8$ \\
\hline $100-<500$ & $11 \cdot 5$ & $(0-100)$ & $45 \cdot 1$ & $9 \cdot 4$ & $(0-40 \cdot 0)$ & $45 \cdot 6$ & $20 \cdot 0$ & $(0-100)$ & $46 \cdot 4$ & $10 \cdot 1$ & $(0-80 \cdot 0)$ & $43 \cdot 6$ & $9 \cdot 1$ & $(0-66 \cdot 1)$ & $43 \cdot 7$ \\
\hline $500-<1000$ & $5 \cdot 6$ & $(0-100)$ & $20 \cdot 3$ & $7 \cdot 3$ & $(0-23 \cdot 7)$ & $21 \cdot 3$ & $20 \cdot 0$ & $(0 \cdot 4-80 \cdot 0)$ & $7 \cdot 7$ & $4 \cdot 5$ & $(0-81 \cdot 1)$ & $19 \cdot 7$ & $4 \cdot 3$ & $(0-100)$ & $42 \cdot 3$ \\
\hline $1000-<1500$ & $5 \cdot 0$ & $(0-11 \cdot 1)$ & $1 \cdot 0$ & $0 \cdot 0$ & $(0-5 \cdot 0)$ & $1 \cdot 8$ & - & $(-)$ & - & - & $(-)$ & - & $6 \cdot 4$ & $(0-11 \cdot 1)$ & $1 \cdot 0$ \\
\hline 1500-1838 & $12 \cdot 3$ & $(12 \cdot 3-12 \cdot 3)$ & $0 \cdot 1$ & - & $(-)$ & - & - & $(-)$ & - & - & $(-)$ & - & $12 \cdot 3$ & $(12 \cdot 3-12 \cdot 3)$ & $0 \cdot 1$ \\
\hline \multicolumn{16}{|l|}{ Diagnostic } \\
\hline IFAT & $11 \cdot 3$ & $(0-100)$ & $76 \cdot 6$ & $4 \cdot 8$ & $(0-43 \cdot 3)$ & $64 \cdot 5$ & $18 \cdot 4$ & $(0-100)$ & $96 \cdot 6$ & $8 \cdot 3$ & $(0-39 \cdot 8)$ & $66 \cdot 5$ & $4 \cdot 4$ & (0-100) & $59 \cdot 6$ \\
\hline ELISA & $11 \cdot 1$ & $(0-100)$ & $8 \cdot 4$ & $11 \cdot 1$ & $(0-27 \cdot 6)$ & $34 \cdot 9$ & $24 \cdot 4$ & $(7 \cdot 1-100)$ & $1 \cdot 1$ & $6 \cdot 5$ & $(6 \cdot 5-6 \cdot 5)$ & $0 \cdot 5$ & $13 \cdot 0$ & $(0-34 \cdot 1)$ & $7 \cdot 5$ \\
\hline Dot ELISA & $7 \cdot 7$ & $(0-36 \cdot 4)$ & $7 \cdot 3$ & - & $(一)$ & - & - & $(-)$ & - & - & $(-)$ & - & $7 \cdot 7$ & $(0-36 \cdot 4)$ & $32 \cdot 4$ \\
\hline DAT & $6 \cdot 6$ & $(0-81 \cdot 1)$ & $5 \cdot 8$ & $25 \cdot 7$ & $(25 \cdot 7-25 \cdot 7)$ & $0 \cdot 6$ & - & $(-)$ & - & $6 \cdot 3$ & $(0-81 \cdot 1)$ & $28 \cdot 7$ & - & $(-)$ & $0 \cdot 0$ \\
\hline Other $^{\mathrm{b}}$ & $4 \cdot 0$ & $(0-66 \cdot 1)$ & 1.9 & - & $(-)$ & - & $1 \cdot 6$ & $(0-37 \cdot 1)$ & $2 \cdot 4$ & $6 \cdot 1$ & $(0-15 \cdot 8)$ & $4 \cdot 3$ & $66 \cdot 1$ & $(66 \cdot 1-66 \cdot 1)$ & $0 \cdot 5$ \\
\hline \multirow[t]{2}{*}{ No. dogs tested } & \multicolumn{3}{|c|}{ All countries $(n=504369)$} & \multicolumn{3}{|c|}{ France $(n=39259)$} & \multicolumn{3}{|c|}{ Italy $(n=423831)$} & \multicolumn{3}{|c|}{ Portugal $(\mathrm{n}=15896)$} & \multicolumn{3}{|c|}{ Spain $(n=25383)$} \\
\hline & Median & $\begin{array}{l}\text { (Interquartile } \\
\text { Range) }\end{array}$ & $\%^{\mathrm{c}}$ & Median & $\begin{array}{l}\text { (Interquartile } \\
\text { Range) }\end{array}$ & $\%^{\mathrm{c}}$ & Median & $\begin{array}{l}\text { (Interquartile } \\
\text { Range) }\end{array}$ & $\%^{\mathrm{c}}$ & Median & $\begin{array}{l}\text { (Interquartile } \\
\text { Range) }\end{array}$ & $\%^{\mathrm{c}}$ & Median & $\begin{array}{l}\text { (Interquartile } \\
\text { Range) }\end{array}$ & $\%^{\mathrm{c}}$ \\
\hline $\begin{array}{l}\text { No. dogs tested } \\
\text { /survey }\end{array}$ & 67 & $(31-209)$ & 100 & 60 & $(32-112)$ & $7 \cdot 8$ & 168 & $(43-612)$ & $84 \cdot 0$ & 52 & $(34-104)$ & $3 \cdot 2$ & 39 & $(20-93)$ & $5 \cdot 0$ \\
\hline
\end{tabular}

a Percentage of surveys out of the total of surveys in all countries. Percentages for categories total $100 \%$, except for altitude because this was not found for 11 surveys (5 in France, 4 in Italy and 2 in Portugal).

b Including CIE, IFAT and CIE, LST. [IFAT: Indirect Fluorescence Antibody Test. ELISA: Enzyme-Linked Immunosorbent Assay. DAT: Direct Agglutination Test. CIE: Counterimmunoelectrophoresis. LST: Leishmanin Skin Test].

${ }^{c}$ Percentage of dogs tested calculated out of the total of dogs tested in all countries. 


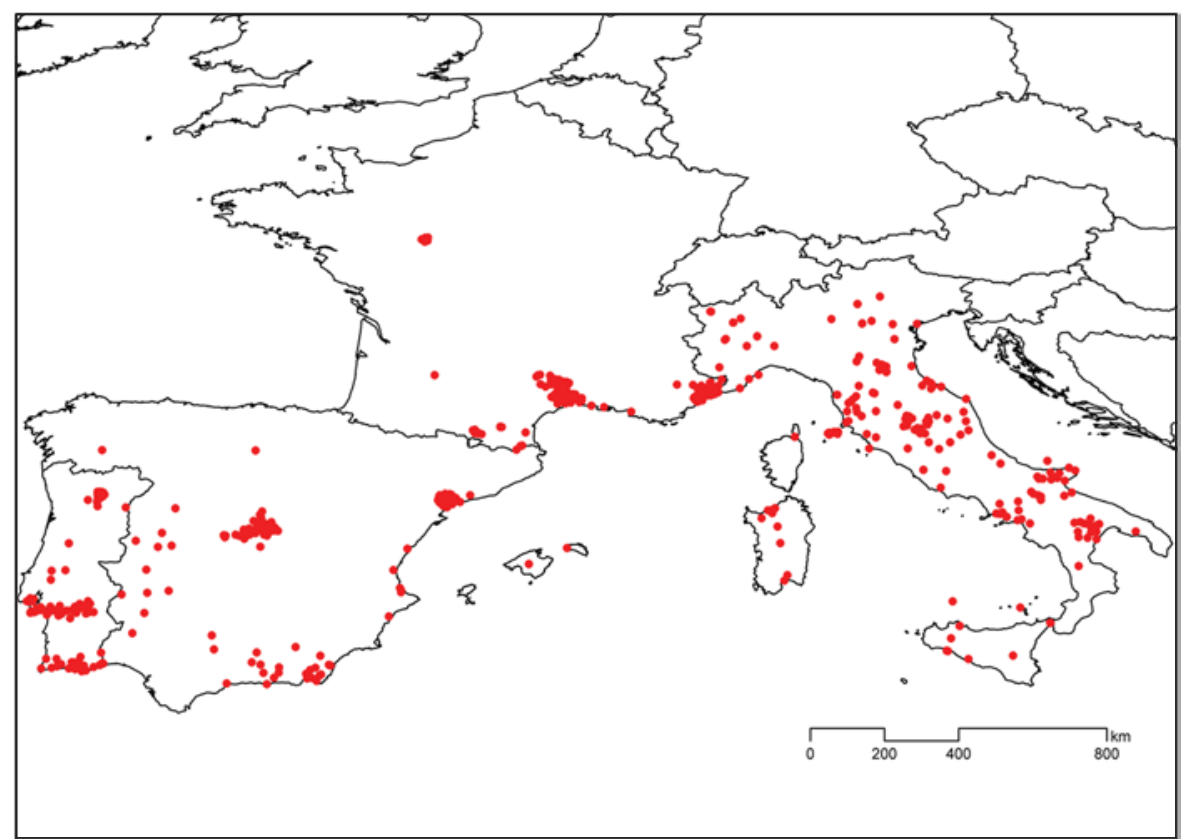

Fig. 1. Distribution of all 947 serological surveys of canine leishmaniasis included in the analyses and modelling of its seroprevalence.

model. However, diagnostic method was not retained, because it did not substantially improve the accuracy of the model predictions. Semivariograms were estimated on the basis of both observed prevalence and Pearson residuals from multivariate models, to investigate the removal of spatial trends potentially related to environmental variables. Both analyses produced unbounded semivariograms (not shown) in which semivariance increased steadily with increasing distance (spatial lags) between observations. In the absence of any clear spatial structure in the prevalence data, modelling was restricted to a non-spatial regression analysis. The final model equation was: CanL seroprevalence $=(\exp (\mathrm{p}) /(1+$ $\exp (\mathrm{p})) \times 100 ;$ where $\mathrm{p}=-2 \cdot 67413-0.5203425 \times$ country_2 $-1.486901 \times$ country_3 $-1.627514 \times$ country $4+0.0015933 \times$ altitude $-8 \cdot 76 \times 10^{-7} \times$ $(\text { altitude })^{2}+0 \cdot 1632941 \times \operatorname{LSTNmn}-0 \cdot 0167179 \times$ $(\mathrm{LSTNmn})^{2}+4 \cdot 346963 \times \mathrm{LSTNa} 3-2 \cdot 562916 \times$ $(\mathrm{LSTNa} 3)^{2}+0 \cdot 0874507 \times 1 / \sqrt{ } \mathrm{EVIa} 1+0 \cdot 136483 \times$ EVIp1 - 0.0361457 × (EVIp1 $)^{2}-0 \cdot 1942139 \times$ (1/EVIp3); and where country_2, country_3 and country_4 were dummy variables for Italy, Portugal and Spain, respectively.

France-only model. Following the same method, the only variables remaining in the final multivariate model were altitude, LSTNmn, amplitude of the biannual cycle of the night-time land surface temperature (LSTNa2), LSTNa3, amplitude of the annual cycle of middle infrared reflectance (MIRa1) and amplitude of the tri-annual cycle of middle infrared reflectance (MIRa3) (Table 4). The final model equation was: $\mathrm{CanL}$ seroprevalence $=(\exp (\mathrm{p}) /$ $(1+\exp (\mathrm{p})) \times 100 ;$ where $\mathrm{p}=-5 \cdot 849738+0 \cdot 0526301 \times$ $\sqrt{ }$ altitude $+32 \cdot 16849 \times$ MIRa1 $-129 \cdot 4284 \times$ MIRa3 + $1 \cdot 429675 \times 1 / \sqrt{ } \mathrm{LSTNa} 2+1 \cdot 130128 \times \mathrm{LSTNa} 3+$ $0 \cdot 2185278 \times$ LSTNmn.

\section{Validation of four-country model}

This was performed with 931 surveys, because at least 1 of the environmental variables in the model was missing for 16 records ( 5 in France, 8 in Italy and 3 in Portugal).

Predicting point-values of CanL seroprevalence: The global correlation coefficient (r) between the predicted and observed seroprevalences was $0 \cdot 341$, and the weakness of this linear agreement was clear in a scatter plot. Predicted seroprevalences were concentrated at $\leqslant 40 \%$, while observed seroprevalences ranged from 0 to $100 \%$. The overall fit did not improve significantly $(r=0 \cdot 352)$ if only the observed seroprevalences $\leqslant 40 \%$ ( $95 \%$ of records) were considered. The country level correlation was considerably stronger for Italy $(r=0.432)$, which had about twice as many surveys as the other 3 countries $(\mathrm{r}=0 \cdot 233-0 \cdot 262)$.

Predicting endemicity classes of CanL seroprevalence: When comparing the model predictions with the observed seroprevalences in the 931 surveys, $41 \%$ of the records were correctly classified to 1 of the 3 endemicity classes (Italy: 48\%; Spain: 40\%; Portugal: $37 \%$; France: $32 \%$ ). Only $5 \cdot 5 \%$ were misclassified to a non-adjacent class (Italy: $3 \cdot 8 \%$; Spain: $4 \cdot 2 \%$; Portugal: $2 \cdot 2 \%$; France: $14 \cdot 6 \%$ ). Overall AUC statistics for the two extreme seroprevalence classes $(<5 \%,>20 \%)$ exceeded the 0.7 threshold for fair to 
Table 2. Univariate binomial logistic regression analyses to test associations between seroprevalence of canine leishmaniasis in the four countries and each potential explanatory variable

\begin{tabular}{|c|c|c|c|c|c|c|}
\hline Variable & & No./947 & Range & Odds Ratio & $\begin{array}{l}\text { (95\% confidence } \\
\text { intervals) }\end{array}$ & $P$-value \\
\hline \multirow[t]{4}{*}{ Country } & France & 169 & & $1 \cdot 00$ & & \multirow{4}{*}{$<0 \cdot 00001^{*}$} \\
\hline & Italy & 377 & & $1 \cdot 02$ & $(0 \cdot 689-1 \cdot 518)$ & \\
\hline & Portugal & 188 & & $0 \cdot 38$ & $(0 \cdot 247-0 \cdot 571)$ & \\
\hline & Spain & 213 & & $0 \cdot 34$ & $(0 \cdot 221-0 \cdot 519)$ & \\
\hline \multirow[t]{5}{*}{ Diagnostic } & Dot ELISA & 69 & & $1 \cdot 00$ & & \multirow[t]{5}{*}{$<0 \cdot 00001 *$} \\
\hline & IFAT & 725 & & $3 \cdot 91$ & $(2 \cdot 331-6 \cdot 569)$ & \\
\hline & ELISA & 80 & & $1 \cdot 56$ & $(0 \cdot 910-2 \cdot 666)$ & \\
\hline & DAT & 55 & & $1 \cdot 12$ & $(0 \cdot 620-2 \cdot 014)$ & \\
\hline & Other (Table 1) & 18 & & $0 \cdot 21$ & $(0 \cdot 118-0 \cdot 392)$ & \\
\hline Surv. date & & 947 & $(1971-2006)$ & $1 \cdot 02$ & $(1 \cdot 001-1 \cdot 047)$ & $0 \cdot 044 *$ \\
\hline Latitude & & 947 & $(35 \cdot 5011-47 \cdot 5267)$ & $0 \cdot 90$ & $(0 \cdot 840-0 \cdot 966)$ & $0 \cdot 004 *$ \\
\hline Altitude & & 936 & $(1-1838)$ & $1 \cdot 00$ & $(0 \cdot 999-1 \cdot 000)$ & $0 \cdot 074 *$ \\
\hline $\mathrm{MIR}^{\mathrm{a}}$ & MIRa0 & 947 & $(0-0 \cdot 243)$ & $2 \cdot 34$ & $(0 \cdot 105-52 \cdot 276)$ & $0 \cdot 591$ \\
\hline (Middle & MIRa1 & 947 & $(0-0 \cdot 104)$ & $20 \cdot 76$ & $(0 \cdot 006-73975 \cdot 1)$ & $0 \cdot 467$ \\
\hline Infrared & MIRa2 & 947 & $(0-0 \cdot 034)$ & $8 \cdot 36 \times 10^{-11}$ & $\left(3 \cdot 12 \times 10^{-26}-2 \cdot 24 \times 10^{5}\right)$ & $0 \cdot 200$ \\
\hline \multirow[t]{6}{*}{ Reflectance) } & MIRa3 & 945 & $(0-0 \cdot 017)$ & $8 \cdot 87 \times 10^{8}$ & $\left(1.11 \times 10^{-8}-7 \cdot 07 \times 10^{25}\right)$ & $0 \cdot 299$ \\
\hline & MIRmn & 947 & $(0-0 \cdot 187)$ & $0 \cdot 97$ & $(0 \cdot 011-86 \cdot 242)$ & $0 \cdot 990$ \\
\hline & MIRmx & 947 & $(0-0 \cdot 317)$ & $2 \cdot 84$ & $(0 \cdot 277-29 \cdot 118)$ & $0 \cdot 380$ \\
\hline & MIRp1 & 947 & $(0-7 \cdot 620)$ & $1 \cdot 32$ & $(1 \cdot 097-1 \cdot 580)$ & $0.003 *$ \\
\hline & MIRp2 & 947 & $(0-5 \cdot 710)$ & $1 \cdot 01$ & $(0.959-1.069)$ & $0 \cdot 658$ \\
\hline & MIRp3 & 947 & $(0-3 \cdot 960)$ & $1 \cdot 02$ & $(0 \cdot 868-1 \cdot 201)$ & $0 \cdot 805$ \\
\hline \multirow{9}{*}{$\begin{array}{l}\mathrm{LSTD}^{\mathrm{a}} \\
\text { (Day-time } \\
\text { land } \\
\text { surface } \\
\text { temperature) }\end{array}$} & LSTDa0 & 938 & $(11 \cdot 96-29 \cdot 58)$ & $1 \cdot 04$ & $(0 \cdot 985-1 \cdot 102)$ & $0 \cdot 153$ \\
\hline & LSTDa1 & 941 & $(5 \cdot 48-17 \cdot 36)$ & $0 \cdot 88$ & $(0 \cdot 822-0 \cdot 935)$ & $0 \cdot 0001 *$ \\
\hline & LSTDa2 & 941 & $(0 \cdot 20-3 \cdot 08)$ & $0 \cdot 73$ & $(0 \cdot 565-0 \cdot 950)$ & $0 \cdot 019^{*}$ \\
\hline & LSTDa3 & 941 & $(0 \cdot 06-1 \cdot 78)$ & $1 \cdot 09$ & $(0 \cdot 652-1 \cdot 820)$ & $0 \cdot 744$ \\
\hline & LSTDmn & 940 & $(-15 \cdot 22-17 \cdot 18)$ & $1 \cdot 15$ & $(1 \cdot 096-1 \cdot 199)$ & $<0 \cdot 00001 *$ \\
\hline & LSTDmx & 938 & $(22 \cdot 56-46 \cdot 08)$ & $0 \cdot 98$ & $(0 \cdot 946-1 \cdot 011)$ & $0 \cdot 187$ \\
\hline & LSTDp1 & 940 & $(6 \cdot 07-7 \cdot 00)$ & $8 \cdot 15$ & $(4 \cdot 070-16 \cdot 321)$ & $<0 \cdot 00001 *$ \\
\hline & LSTDp2 & 941 & $(0 \cdot 61-3 \cdot 10)$ & $0 \cdot 62$ & $(0 \cdot 442-0 \cdot 856)$ & $0 \cdot 004 *$ \\
\hline & LSTDp3 & 941 & $(0 \cdot 03-3 \cdot 98)$ & $1 \cdot 20$ & $(1 \cdot 017-1 \cdot 428)$ & $0 \cdot 0313 *$ \\
\hline \multirow{9}{*}{$\begin{array}{l}\mathrm{LSTN}^{\mathrm{a}} \\
\text { (Night-time } \\
\text { land } \\
\text { surface } \\
\text { temperature) }\end{array}$} & LSTNa0 & 938 & $(3 \cdot 98-15 \cdot 56)$ & $1 \cdot 10$ & $(1 \cdot 045-1 \cdot 169)$ & $0 \cdot 0005^{*}$ \\
\hline & LSTNa1 & 941 & $(5 \cdot 10-11 \cdot 80)$ & $0 \cdot 83$ & $(0.742-0.919)$ & $0 \cdot 0004 *$ \\
\hline & LSTNa2 & 941 & $(0 \cdot 10-2 \cdot 24)$ & $1 \cdot 07$ & $(0 \cdot 830-1 \cdot 381)$ & $0 \cdot 599$ \\
\hline & LSTNa3 & 941 & $(0 \cdot 04-1 \cdot 64)$ & $1 \cdot 86$ & $(1 \cdot 166-2 \cdot 967)$ & $0 \cdot 009^{*}$ \\
\hline & LSTNmn & 938 & $(-7 \cdot 40-9 \cdot 90)$ & $1 \cdot 11$ & $(1 \cdot 071-1 \cdot 156)$ & $<0 \cdot 00001 *$ \\
\hline & LSTNmx & 938 & $(13 \cdot 76-26 \cdot 78)$ & $1 \cdot 07$ & $(1 \cdot 010-1 \cdot 129)$ & $0 \cdot 021 *$ \\
\hline & LSTNp1 & 940 & $(6 \cdot 41-7 \cdot 26)$ & $28 \cdot 73$ & $(11 \cdot 335-72 \cdot 810)$ & $<0 \cdot 00001 *$ \\
\hline & LSTNp2 & 941 & $(0 \cdot 03-5 \cdot 86)$ & $0 \cdot 51$ & $(0 \cdot 333-0 \cdot 790)$ & $0 \cdot 003^{*}$ \\
\hline & LSTNp3 & 941 & $(0 \cdot 14-2 \cdot 91)$ & $0 \cdot 98$ & $(0 \cdot 745-1 \cdot 292)$ & $0 \cdot 891$ \\
\hline \multirow{9}{*}{$\begin{array}{l}\text { EVI }^{\mathrm{a}} \\
\text { (Enhanced } \\
\text { vegetation } \\
\text { Index) }\end{array}$} & EVIa0 & 945 & $(-0 \cdot 035-0 \cdot 430)$ & $0 \cdot 18$ & $(0 \cdot 056-0 \cdot 610)$ & $0 \cdot 006 *$ \\
\hline & EVIa1 & 945 & $(0 \cdot 002-0 \cdot 247)$ & $0 \cdot 01$ & $\left(6.86 \times 10^{-5}-0.792\right)$ & $0 \cdot 040^{*}$ \\
\hline & EVIa2 & 922 & $(0 \cdot 002-0 \cdot 085)$ & $0 \cdot 01$ & $\left(2.32 \times 10^{-7}-6.93 \times 10^{-2}\right)$ & $0 \cdot 433$ \\
\hline & EVIa3 & 944 & $(0 \cdot 001-0 \cdot 054)$ & $5 \cdot 27 \times 10^{-6}$ & $\left(5 \cdot 00 \times 10^{-16}-5.55 \times 10^{4}\right)$ & $0 \cdot 302$ \\
\hline & EVImn & 945 & $(-0 \cdot 093-0 \cdot 371)$ & $0 \cdot 24$ & $(0 \cdot 044-1 \cdot 286)$ & $0.095 *$ \\
\hline & EVImx & 945 & $(0 \cdot 005-0 \cdot 653)$ & $0 \cdot 34$ & $(0 \cdot 129-0 \cdot 911)$ & $0 \cdot 032 *$ \\
\hline & EVIp1 & 945 & $(0 \cdot 530-10 \cdot 530)$ & $0 \cdot 90$ & $(0 \cdot 827-0 \cdot 974)$ & $0 \cdot 010^{*}$ \\
\hline & EVIp2 & 945 & $(0 \cdot 040-5 \cdot 920)$ & $0 \cdot 83$ & $(0 \cdot 747-0 \cdot 913)$ & $0 \cdot 0002 *$ \\
\hline & EVIp3 & 945 & $(0 \cdot 040-3 \cdot 990)$ & $1 \cdot 21$ & $(1 \cdot 078-1 \cdot 349)$ & $0 \cdot 001 *$ \\
\hline \multirow[t]{8}{*}{ Climate $^{\mathrm{b}}$} & DTR summer & 925 & $(5 \cdot 98-16 \cdot 20)$ & $0 \cdot 85$ & $(0 \cdot 817-0 \cdot 893)$ & $<0 \cdot 00001 *$ \\
\hline & DTR winter & 925 & $(4 \cdot 79-11 \cdot 50)$ & $0 \cdot 84$ & $(0 \cdot 789-0 \cdot 897)$ & $<0 \cdot 00001 *$ \\
\hline & TMP summer & 925 & $(11 \cdot 36-23 \cdot 50)$ & $1 \cdot 18$ & $(1 \cdot 070-1 \cdot 295)$ & $0 \cdot 001 *$ \\
\hline & TMP winter & 925 & $(0 \cdot 63-14 \cdot 77)$ & $1 \cdot 17$ & $(1 \cdot 108-1 \cdot 244)$ & $<0 \cdot 00001 *$ \\
\hline & PRE summer & 925 & $(9 \cdot 12-107 \cdot 98)$ & $0 \cdot 98$ & $(0 \cdot 971-0.988)$ & $<0 \cdot 00001 *$ \\
\hline & PRE winter & 925 & $(28 \cdot 30-146 \cdot 40)$ & $1 \cdot 00$ & $(0 \cdot 989-1 \cdot 001)$ & $0 \cdot 126$ \\
\hline & REH summer & 925 & $(38 \cdot 30-62 \cdot 42)$ & $1 \cdot 05$ & $(1 \cdot 031-1 \cdot 078)$ & $<0 \cdot 00001 *$ \\
\hline & REH winter & 925 & $(61 \cdot 90-86 \cdot 20)$ & $1 \cdot 01$ & $(0 \cdot 951-1 \cdot 070)$ & $0 \cdot 767$ \\
\hline
\end{tabular}

a The last 2 characters denote the output from Fourier processing:-a0: mean, mn: minimum, mx: maximum, a1: amplitude of annual cycle, a2: amplitude of bi-annual cycle, a3: amplitude of tri-annual cycle, p1: phase of annual cycle, p2: phase of bi-annual cycle, p3: phase of tri-annual cycle; ${ }^{\mathrm{b}}$ DTR: diurnal temperature range, TMP: mean temperature, PRE: rainfall, REH: relative humidity, summer: May-September, winter: October-April; * $P<0 \cdot 1$ in the univariate analysis. 
Table 3. Multivariate binomial logistic regression analysis of the associations between seroprevalence of canine leishmaniasis and each explanatory variable (or risk factor) for the four-country model

\begin{tabular}{|c|c|c|c|c|c|c|c|c|}
\hline $\begin{array}{l}\text { Explanatory } \\
\text { variable }\end{array}$ & Category & Ref. point & $\begin{array}{l}\text { No. dogs } \\
\text { tested }\end{array}$ & $\begin{array}{l}\text { No. } \\
\text { surveys }\end{array}$ & $\begin{array}{l}\text { Mean } \\
\text { prevalence }\end{array}$ & $\begin{array}{l}\text { (Standard } \\
\text { deviation) }\end{array}$ & $\mathrm{OR}^{\mathrm{b}}$ & $(95 \% \mathrm{Cl})^{\mathrm{c}}$ \\
\hline \multirow[t]{4}{*}{ Country } & France & & 39259 & 169 & $10 \cdot 3$ & $(9 \cdot 9)$ & $1 \cdot 00$ & \\
\hline & Italy & & 423831 & 377 & $20 \cdot 6$ & $(17 \cdot 8)$ & 0.59 & $(0 \cdot 40-0 \cdot 87)$ \\
\hline & Portugal & & 15896 & 188 & $12 \cdot 0$ & $(13 \cdot 6)$ & $0 \cdot 23$ & $(0 \cdot 14-0 \cdot 37)$ \\
\hline & Spain & & 25383 & 213 & $9 \cdot 5$ & $(12 \cdot 0)$ & $0 \cdot 20$ & $(0 \cdot 13-0 \cdot 31)$ \\
\hline \multirow[t]{4}{*}{ Altitude } & $1-99$ & 50 & 390292 & 307 & $15 \cdot 7$ & $(15 \cdot 3)$ & $1 \cdot 00$ & \\
\hline & $100-499$ & 300 & 74578 & 427 & $15 \cdot 8$ & $(15 \cdot 5)$ & $1 \cdot 38$ & $(1 \cdot 18-1 \cdot 61)$ \\
\hline & 500-999 & 750 & 32297 & 192 & $10 \cdot 6$ & $(15 \cdot 1)$ & $1 \cdot 87$ & $(1 \cdot 27-2 \cdot 74)$ \\
\hline & $1000-1838$ & 1400 & 1906 & 10 & $5 \cdot 3$ & $(4.9)$ & $1 \cdot 55$ & $(0 \cdot 74-3 \cdot 25)$ \\
\hline $\operatorname{LSTNmn}^{\mathrm{a}}$ & $-7 \cdot 40-0 \cdot 34$ & $-4 \cdot 00$ & 36642 & 191 & $11 \cdot 7$ & $(16 \cdot 0)$ & $1 \cdot 00$ & \\
\hline (Night-time & $-0 \cdot 33-1 \cdot 10$ & $0 \cdot 40$ & 148263 & 183 & $12 \cdot 0$ & $(14 \cdot 6)$ & $2 \cdot 67$ & $(1 \cdot 89-3 \cdot 78)$ \\
\hline land surface & $1 \cdot 11-2 \cdot 30$ & $1 \cdot 70$ & 78823 & 190 & $18 \cdot 3$ & $(17 \cdot 2)$ & $3 \cdot 16$ & $(2 \cdot 05-4 \cdot 88)$ \\
\hline \multirow[t]{2}{*}{ temperature) } & $2 \cdot 31-4 \cdot 40$ & $3 \cdot 50$ & 142838 & 188 & $14 \cdot 5$ & $(13 \cdot 8)$ & $3 \cdot 62$ & $(2 \cdot 14-6 \cdot 15)$ \\
\hline & $4 \cdot 41-9 \cdot 90$ & $7 \cdot 00$ & 92977 & 186 & $15 \cdot 4$ & $(13 \cdot 7)$ & $3 \cdot 47$ & $(1 \cdot 89-6 \cdot 38)$ \\
\hline $\operatorname{LSTNa}^{\mathrm{a}}$ & $0 \cdot 04-0 \cdot 30$ & $0 \cdot 15$ & 16231 & 211 & $10 \cdot 1$ & $(10 \cdot 0)$ & $1 \cdot 00$ & \\
\hline (Night-time & $0 \cdot 31-0 \cdot 37$ & $0 \cdot 35$ & 20283 & 156 & $11 \cdot 3$ & $(13 \cdot 5)$ & $1 \cdot 85$ & $(1 \cdot 27-2 \cdot 68)$ \\
\hline land surface & $0 \cdot 38-0 \cdot 50$ & $0 \cdot 45$ & 43745 & 206 & $12 \cdot 0$ & $(14 \cdot 0)$ & $2 \cdot 32$ & $(1 \cdot 40-3 \cdot 87)$ \\
\hline \multirow[t]{2}{*}{ temperature) } & $0 \cdot 51-1 \cdot 00$ & $0 \cdot 75$ & 354105 & 338 & $20 \cdot 1$ & $(17 \cdot 9)$ & $3 \cdot 40$ & $(1 \cdot 78-6 \cdot 49)$ \\
\hline & $1 \cdot 01-1 \cdot 64$ & $1 \cdot 15$ & 65376 & 30 & $16 \cdot 1$ & $(15 \cdot 5)$ & $2 \cdot 76$ & $(2 \cdot 52-3 \cdot 02)$ \\
\hline EVIa1 $^{\mathrm{a}}$ & $0 \cdot 002-0 \cdot 025$ & $0 \cdot 015$ & 330482 & 234 & $15 \cdot 8$ & $(15 \cdot 0)$ & $1 \cdot 00$ & \\
\hline (Enhanced & $0 \cdot 026-0.050$ & $0 \cdot 030$ & 104380 & 322 & $14 \cdot 9$ & $(15 \cdot 7)$ & $0 \cdot 81$ & $(0 \cdot 71-0 \cdot 98)$ \\
\hline vegetation & $0 \cdot 051-0 \cdot 075$ & $0 \cdot 045$ & 22709 & 179 & $15 \cdot 2$ & $(16 \cdot 8)$ & $0 \cdot 74$ & $(0 \cdot 61-0.95)$ \\
\hline \multirow[t]{2}{*}{ Index) } & $0 \cdot 076-0 \cdot 100$ & $0 \cdot 065$ & 21528 & 98 & $14 \cdot 0$ & $(14 \cdot 8)$ & $0 \cdot 69$ & $(0.54-0.93)$ \\
\hline & $0 \cdot 101-0 \cdot 247$ & $0 \cdot 165$ & 25097 & 112 & $10 \cdot 0$ & $(12 \cdot 5)$ & $0 \cdot 61$ & $(0 \cdot 44-0 \cdot 89)$ \\
\hline EVIp1 ${ }^{a}$ & $0 \cdot 53-3 \cdot 00$ & $1 \cdot 5$ & 18641 & 180 & $14 \cdot 4$ & $(17 \cdot 3)$ & $1 \cdot 00$ & \\
\hline (Enhanced & $3 \cdot 01-4 \cdot 50$ & $3 \cdot 5$ & 28261 & 91 & $15 \cdot 4$ & $(16 \cdot 0)$ & 0.92 & $(0 \cdot 61-1 \cdot 38)$ \\
\hline vegetation & $4 \cdot 51-6 \cdot 00$ & $5 \cdot 5$ & 186709 & 280 & $15 \cdot 6$ & $(14 \cdot 7)$ & $0 \cdot 63$ & $(0 \cdot 36-1 \cdot 10)$ \\
\hline \multirow[t]{2}{*}{ Index) } & $6 \cdot 01-7 \cdot 00$ & $6 \cdot 5$ & 268213 & 362 & 13.9 & $(15 \cdot 0)$ & $0 \cdot 47$ & $(0.28-0.79)$ \\
\hline & $7 \cdot 01-10 \cdot 53$ & $8 \cdot 5$ & 2372 & 32 & $10 \cdot 5$ & $(10 \cdot 8)$ & $0 \cdot 21$ & $(0 \cdot 16-0 \cdot 27)$ \\
\hline $\mathrm{EVIp}^{\mathrm{a}}$ & $0 \cdot 40-0 \cdot 70$ & $0 \cdot 50$ & 89441 & 191 & $14 \cdot 2$ & $(16 \cdot 5)$ & $1 \cdot 00$ & \\
\hline (Enhanced & $0 \cdot 71-1 \cdot 00$ & $0 \cdot 80$ & 83086 & 192 & $15 \cdot 9$ & $(14 \cdot 1)$ & $1 \cdot 16$ & $(1 \cdot 11-1 \cdot 21)$ \\
\hline vegetation & $1 \cdot 01-1 \cdot 40$ & $1 \cdot 20$ & 123799 & 173 & $12 \cdot 4$ & $(13.9)$ & $1 \cdot 25$ & $(1 \cdot 18-1 \cdot 34)$ \\
\hline \multirow[t]{2}{*}{ Index) } & $1 \cdot 41-3 \cdot 00$ & $2 \cdot 20$ & 159632 & 205 & $15 \cdot 9$ & $(15 \cdot 4)$ & $1 \cdot 35$ & $(1 \cdot 24-1 \cdot 47)$ \\
\hline & $3 \cdot 01-3 \cdot 99$ & $3 \cdot 50$ & 48238 & 184 & $13 \cdot 9$ & $(16 \cdot 5)$ & $1 \cdot 40$ & $(1 \cdot 27-1 \cdot 53)$ \\
\hline
\end{tabular}

a The last 2 characters denote the output from Fourier processing:- mn: minimum, a1: amplitude of annual cycle, a3: amplitude of tri-annual cycle, p1: phase of annual cycle, p3: phase of tri-annual cycle.

b Odds Ratio $(\mathrm{OR})$ was calculated for the reference point of the categories of each variable $\mathrm{X}_{\mathrm{i}}$ from the final model: Log Odds $=-2.67413+\beta_{\mathrm{i}} \mathrm{X}_{\mathrm{i}}$. Where $\beta_{\mathrm{i}} \mathrm{X}_{\mathrm{i}}$ is: for country, $-0.5203425 \times$ country_2 $-1.486901 \times$ country_3 $-1.627514 \times$ country_4 (where the baseline country is France, and country_2, country_3 and country_4 are dummy variables for country $=$ Italy, Portugal or Spain, respectively); for altitude: $0 \cdot 0015933 \times$ altitude $-8 \cdot 76 \times 10^{-\overline{7}} \times(\text { altitude })^{2}$; for LSTNmn:

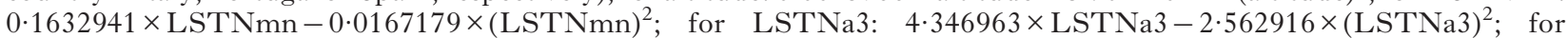
EVIa1: $0 \cdot 0874507 \times 1 / \sqrt{ }$ EVIa1; for EVIp1: $0 \cdot 136483 \times$ EVIp1 - 0.0361457 $\times(\text { EVIp1 })^{2}$; and for EVIp3: $-0 \cdot 1942139 \times$ (1/EVIp3).

c The $95 \%$ confidence interval (CI) was calculated according to Pullan et al. (2008).

good discrimination ( $\mathrm{AUC}=0 \cdot 71$ and $0 \cdot 73$, respectively), but the AUC for the intermediate class (5-20\%) was below the threshold ( $\mathrm{AUC}=0 \cdot 55)$. When looking at the results by country, similar results applied to France and Italy, where the model performed best, while in Portugal and Spain the AUC for all CanL seroprevalence classes was below $0 \cdot 6$.

\section{Validation of France-only model}

This was performed with 164 of the 169 surveys carried out from 1971 to 2006 , because at least 1 of the environmental variables in the model was missing for 5 records.
Predicting point-values of CanL seroprevalence: The global correlation coefficient (r) between the predicted and observed seroprevalences was $0 \cdot 648$. The range of predicted seroprevalences was $2-38 \%$, which is comparable to the observed range of $0-43 \%$.

Predicting endemicity classes of CanL seroprevalence: When comparing the model predictions with the observed seroprevalences in the 164 surveys, $54 \%$ of the records were correctly classified to 1 of the 3 endemicity classes, substantially more than those correctly classified for France $(32 \%)$ by the four-country model. Only $3 \%$ were misclassified to a non-adjacent class, substantially fewer than those misclassified for France (14.6\%) by the four-country 
Table 4. Multivariate binomial logistic regression analysis of the associations between seroprevalence of canine leishmaniasis and each explanatory variable (or risk factor) for the France-only model

\begin{tabular}{|c|c|c|c|c|c|c|c|c|}
\hline $\begin{array}{l}\text { Explanatory } \\
\text { variable }\end{array}$ & Category & Ref. point & $\begin{array}{l}\text { No. dogs } \\
\text { tested }\end{array}$ & $\begin{array}{l}\text { No. } \\
\text { surveys }\end{array}$ & $\begin{array}{l}\text { Mean } \\
\text { prevalence }\end{array}$ & $\begin{array}{l}\text { (Standard } \\
\text { deviation) }\end{array}$ & $\mathrm{OR}^{\mathrm{b}}$ & $(95 \% \mathrm{CI})^{\mathrm{c}}$ \\
\hline \multirow[t]{4}{*}{ Altitude } & $1-99$ & 50 & 30444 & 48 & $13 \cdot 2$ & $(14 \cdot 0)$ & $1 \cdot 00$ & \\
\hline & $100-249$ & 175 & 3247 & 41 & $9 \cdot 8$ & $(7 \cdot 8)$ & $1 \cdot 38$ & $(1 \cdot 15-1 \cdot 67)$ \\
\hline & $250-499$ & 375 & 2751 & 36 & $11 \cdot 1$ & $(7 \cdot 3)$ & 1.91 & $(1 \cdot 31-2 \cdot 78)$ \\
\hline & $500-1838$ & 750 & 2121 & 39 & $6 \cdot 8$ & $(6 \cdot 5)$ & $2 \cdot 91$ & $(1 \cdot 57-5 \cdot 42)$ \\
\hline \multirow{4}{*}{$\begin{array}{l}\text { LSTNmn }^{\text {a }} \\
\text { (Night-time } \\
\text { land surface } \\
\text { temperature) }\end{array}$} & $-5 \cdot 16-0 \cdot 34$ & $-3 \cdot 00$ & 3089 & 48 & $5 \cdot 4$ & $(4 \cdot 9)$ & $1 \cdot 00$ & \\
\hline & $-0 \cdot 33-1 \cdot 10$ & $0 \cdot 40$ & 2660 & 42 & $7 \cdot 4$ & $(7 \cdot 9)$ & $2 \cdot 10$ & $(1 \cdot 34-3 \cdot 31)$ \\
\hline & $1 \cdot 11-2 \cdot 30$ & $1 \cdot 70$ & 3545 & 38 & $11 \cdot 1$ & $(7 \cdot 2)$ & $2 \cdot 79$ & $(1 \cdot 49-5 \cdot 22)$ \\
\hline & $2 \cdot 31-4 \cdot 66$ & $3 \cdot 50$ & 29965 & 41 & $18 \cdot 4$ & $(12 \cdot 7)$ & $4 \cdot 14$ & $(1 \cdot 74-9 \cdot 83)$ \\
\hline \multirow{5}{*}{$\begin{array}{l}\text { LSTNa2 }^{\text {a }} \\
\text { (Night-time } \\
\text { land surface } \\
\text { temperature) }\end{array}$} & $0 \cdot 180-0 \cdot 50$ & $0 \cdot 35$ & 27794 & 32 & $17 \cdot 4$ & $(14 \cdot 4)$ & $1 \cdot 00$ & \\
\hline & $0 \cdot 510-0 \cdot 66$ & $0 \cdot 60$ & 2455 & 28 & $10 \cdot 2$ & $(7 \cdot 4)$ & $0 \cdot 57$ & $(0 \cdot 42-0 \cdot 76)$ \\
\hline & $0 \cdot 661-0 \cdot 80$ & $0 \cdot 75$ & 3195 & 40 & $9 \cdot 7$ & $(8 \cdot 8)$ & $0 \cdot 46$ & $(0 \cdot 31-0 \cdot 69)$ \\
\hline & $0 \cdot 801-1 \cdot 00$ & $0 \cdot 90$ & 2566 & 38 & $6 \cdot 2$ & $(7 \cdot 1)$ & $0 \cdot 40$ & $(0 \cdot 25-0 \cdot 65)$ \\
\hline & $1 \cdot 010-2 \cdot 24$ & $1 \cdot 50$ & 3249 & 31 & $9 \cdot 2$ & $(6 \cdot 5)$ & $0 \cdot 29$ & $(0 \cdot 15-0 \cdot 55)$ \\
\hline \multirow{5}{*}{$\begin{array}{l}\text { LSTNa3 }^{\text {a }} \\
\text { (Night-time } \\
\text { land surface } \\
\text { temperature) }\end{array}$} & $0 \cdot 040-0 \cdot 30$ & $0 \cdot 15$ & 3343 & 38 & $8 \cdot 8$ & $(8 \cdot 9)$ & $1 \cdot 00$ & \\
\hline & $0 \cdot 301-0 \cdot 37$ & $0 \cdot 35$ & 3342 & 39 & $10 \cdot 4$ & $(7 \cdot 3)$ & $1 \cdot 25$ & $(1 \cdot 02-1 \cdot 55)$ \\
\hline & $0 \cdot 371-0 \cdot 50$ & $0 \cdot 45$ & 3221 & 39 & $8 \cdot 5$ & $(6 \cdot 3)$ & $1 \cdot 40$ & $(1 \cdot 02-1 \cdot 92)$ \\
\hline & $0 \cdot 501-0 \cdot 65$ & $0 \cdot 55$ & 28276 & 30 & $18 \cdot 3$ & $(15 \cdot 2)$ & $1 \cdot 57$ & $(1 \cdot 03-2 \cdot 39)$ \\
\hline & $0 \cdot 651-1 \cdot 54$ & $1 \cdot 15$ & 1077 & 23 & $5 \cdot 6$ & $(5 \cdot 3)$ & $3 \cdot 10$ & $(1 \cdot 08-8 \cdot 87)$ \\
\hline \multirow{5}{*}{$\begin{array}{l}\text { MIRa1 } \\
\text { (Middle }^{\text {Midde }} \\
\text { Infrared } \\
\text { reflectance) }\end{array}$} & $0 \cdot 0060-0 \cdot 016$ & $0 \cdot 010$ & 1980 & 35 & $7 \cdot 7$ & $(6 \cdot 8)$ & $1 \cdot 00$ & \\
\hline & $0 \cdot 0161-0 \cdot 019$ & $0 \cdot 017$ & 2932 & 31 & $9 \cdot 3$ & $(6 \cdot 9)$ & $1 \cdot 25$ & $(1 \cdot 08-1 \cdot 46)$ \\
\hline & $0 \cdot 0191-0 \cdot 025$ & $0 \cdot 023$ & 2807 & 35 & $10 \cdot 0$ & $(6 \cdot 7)$ & $1 \cdot 52$ & $(1 \cdot 15-2 \cdot 01)$ \\
\hline & $0 \cdot 0251-0 \cdot 032$ & $0 \cdot 030$ & 3049 & 34 & $9 \cdot 8$ & $(9 \cdot 0)$ & 1.90 & $(1 \cdot 23-2 \cdot 93)$ \\
\hline & $0 \cdot 0321-0 \cdot 040$ & $0 \cdot 036$ & 28491 & 34 & $14 \cdot 9$ & $(15 \cdot 7)$ & $2 \cdot 31$ & $(1 \cdot 31-4 \cdot 05)$ \\
\hline \multirow{5}{*}{$\begin{array}{l}\text { MIRa3 }^{\mathrm{a}} \\
\text { (Middle }^{\text {Infrared }} \\
\text { reflectance) }\end{array}$} & $0 \cdot 0005-0.0014$ & $0 \cdot 001$ & 28139 & 29 & $20 \cdot 3$ & $(13 \cdot 7)$ & $1 \cdot 00$ & \\
\hline & $0 \cdot 0015-0.0026$ & $0 \cdot 002$ & 3461 & 32 & $12 \cdot 6$ & $(8 \cdot 0)$ & $0 \cdot 87$ & $(0 \cdot 78-1 \cdot 00)$ \\
\hline & $0 \cdot 0027-0 \cdot 0042$ & 0.003 & 2800 & 37 & $8 \cdot 0$ & $(5 \cdot 7)$ & 0.77 & $(0 \cdot 60-0.99)$ \\
\hline & $0 \cdot 0043-0 \cdot 0056$ & $0 \cdot 005$ & 2914 & 35 & $6 \cdot 8$ & $(7 \cdot 9)$ & 0.59 & $(0 \cdot 36-0 \cdot 98)$ \\
\hline & $0 \cdot 0057-0 \cdot 0106$ & $0 \cdot 008$ & 1945 & 36 & $6 \cdot 1$ & $(6 \cdot 5)$ & $0 \cdot 40$ & $(0 \cdot 17-0 \cdot 97)$ \\
\hline
\end{tabular}

a The last 2 characters denote the output from Fourier processing: - mn: minimum, a1: amplitude of annual cycle, a2: amplitude of bi-annual cycle, a3: amplitude of tri-annual cycle.

b Odds Ratio (OR) was calculated for the reference point of the categories of each variable Xi from the final model: Log Odds $=-5 \cdot 849738+\beta \mathrm{iXi}$. Where $\beta \mathrm{iX}$ is, for altitude: $0 \cdot 0526301 \times \sqrt{ }$ altitude; for LSTNmn: $0 \cdot 2185278 \times \mathrm{LSTNmn}$; for

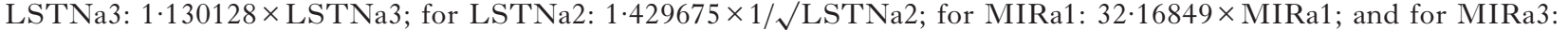
$-129 \cdot 4284 \times$ MIRa3.

c The 95\% confidence interval (CI) was calculated according to Pullan et al. (2008).

model. Overall AUC statistics for the two extreme seroprevalence classes $(<5 \%,>20 \%)$ exceeded the $0 \cdot 7$ threshold for fair to good discrimination $(\mathrm{AUC}=0.77$ and $0 \cdot 81$, respectively), but the AUC for the intermediate class (5-20\%) was below the threshold (AUC $=0 \cdot 64)$.

\section{Risk maps}

The predicted CanL seroprevalence was mapped for the four-country model (Fig. 2) and for the Franceonly model (Fig. 3) using 5 endemicity classes. Pixels were unclassified if their environmental values were outside the range of those in the survey locations. When estimating seroprevalence for the risk maps, a minimum increment was added to each value of EVIp3, EVIa1 and LSTNa2, in order to eliminate any zero values. In the four-country model, EVIp3 and EVIa1 were replaced by EVIp $3+0 \cdot 01$ and EVIa1 + 0.001, respectively; and, in the France-only model, LSTNa2 was replaced by $\mathrm{LSTNa}_{2}+0 \cdot 02$.
Unclassified pixels were relatively few in number for the four-country model, but considerably higher for the France-only model. For the four-country map, a total of $4034(6 \cdot 31 \%)$ pixels were unclassified (Italy: 1969 (14·88\%); Spain: 661 (3·12\%); Portugal: $55(1 \cdot 47 \%))$, with 1711 of these pixels being outside the altitude range of the survey locations in the four countries $(254<1 \mathrm{~m}$ a.s.1.; $1457>1838 \mathrm{~m}$ a.s.1.), and $50 \%$ of the unclassified pixels were located $<1492$ m a.s.l. For France mapped using the fourcountry model, a total of $1349(5 \cdot 24 \%)$ pixels were unclassified, with 575 of these pixels being outside the altitude range of the survey locations in the four countries $(81<1 \mathrm{~m}$ a.s.1.; $494>1838 \mathrm{~m}$ a.s.1. $)$, and $50 \%$ of the unclassified pixels were located $<1457 \mathrm{~m}$ a.s.l. In contrast, for France investigated by the France-only model, a total of 7998 (31.05\%) pixels were unclassified, with 1333 of these pixels being outside the altitude range of the survey locations in France $(81<1 \mathrm{~m}$ a.s.1.; $1252>1193 \mathrm{~m}$ a.s.1.), and 50\% of the unclassified pixels were located $<257 \mathrm{~m}$ a.s.1. 


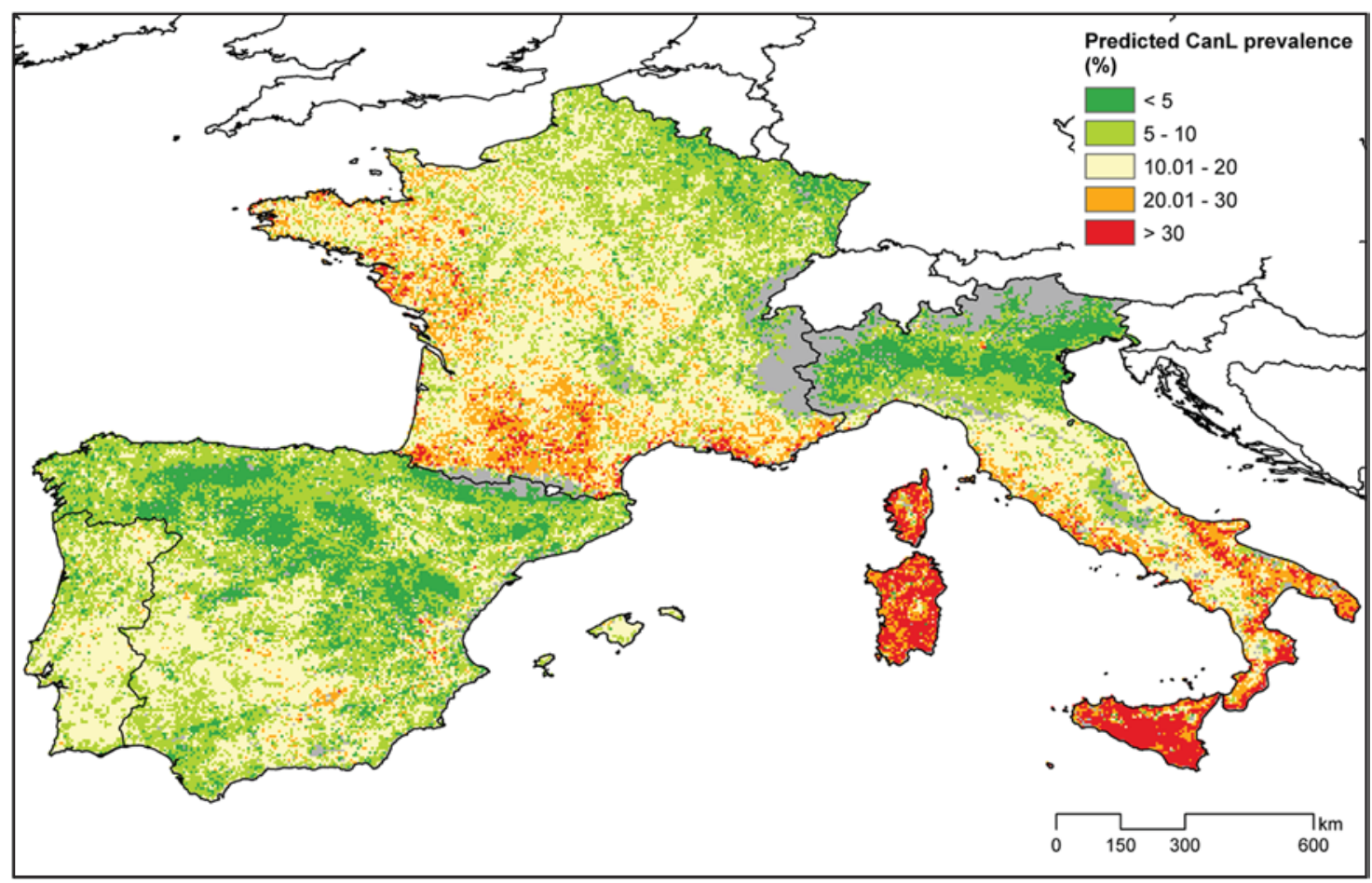

Fig. 2. Risk map for canine leishmaniasis (CanL) in Portugal, Spain, France and Italy based on the four-country model. Predicted CanL seroprevalence was mapped only for the pixels where the predictive environmental values were within the range of those in the survey locations. Other pixels, those outside this mask, are shaded in grey.

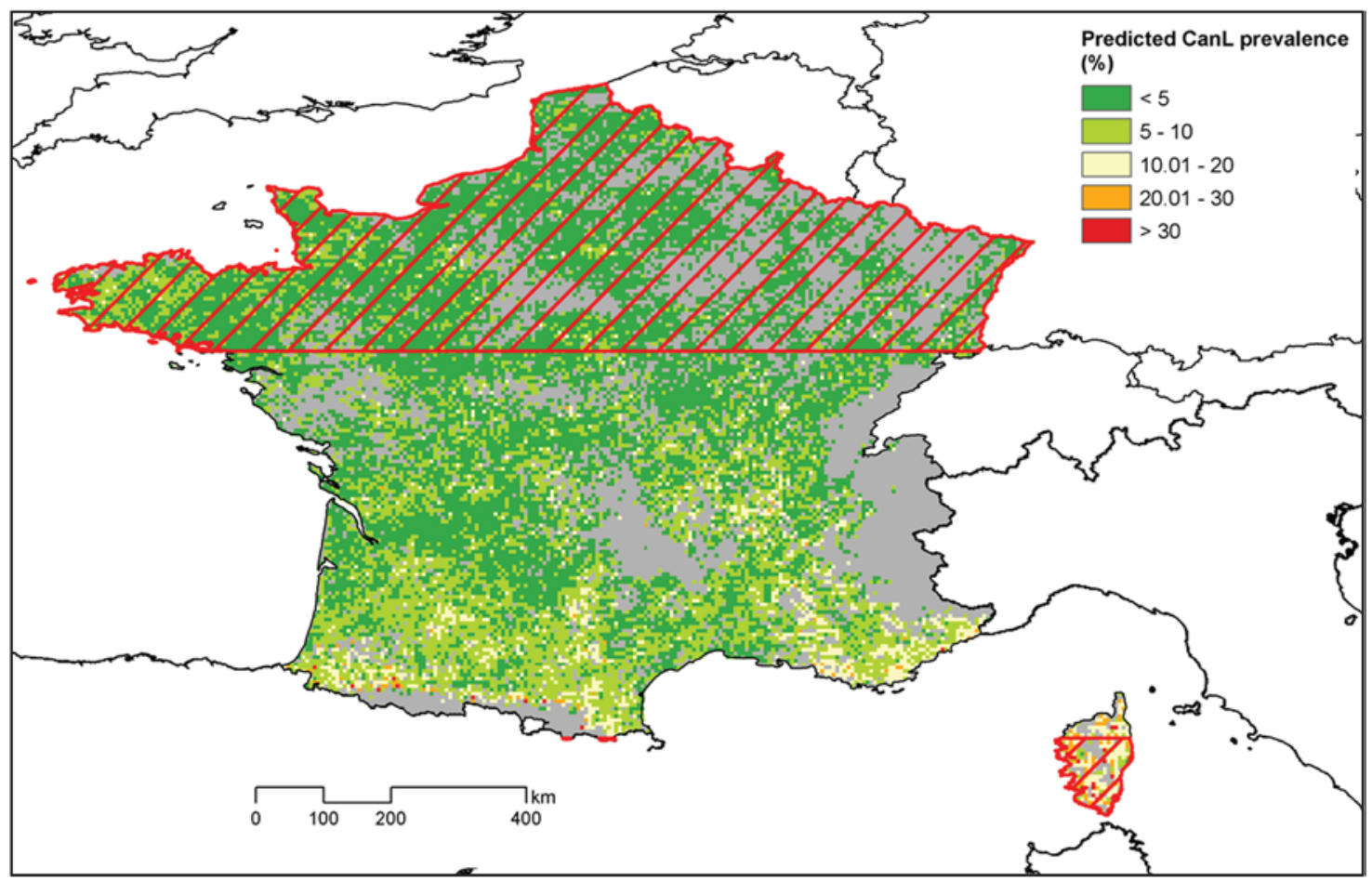

Fig. 3. Risk map for canine leishmaniasis (CanL) in France based on the France-only model. Predicted CanL seroprevalence was mapped only for the pixels where the predictive environmental values were within the range of those in the survey locations. Other pixels, those outside this mask, are shaded in grey. The areas marked by red diagonals show France outside the latitudinal range of all the surveys analysed. Latitude was not a predictive variable. 
For France, overall, there were almost 13 times more pixels not classified at altitudes $\leqslant 1193 \mathrm{~m}$ a.s.l. in the France-only risk map compared with the fourcountry one.

\section{DISCUSSION}

Our database is unique. It is the first time that detailed historical records of CanL in endemic European countries have been collated in this standardized way for such a long period (19652006), and the database is an important resource for future eco-epidemiological analyses at country and regional levels. However, like all such databases, its entries mirror the different histories of European teams working with changing diagnostic techniques and public health priorities (Dujardin et al. 2008), including the period (1990-1998) when epidemiological investigations of ZVL focused on co-infections with HIV. Partly for these reasons, many records were not suitable for our specific analyses, and we hope this finding will prompt better standardization of prospective surveys when cost effective.

Our use of this database in the current report has produced 2 sets of novel findings. One set arises from the construction of the first explicit risk map for CanL in western Mediterranean Europe and consideration of the lessons to be learned from its apparent deficiencies. The second set of findings relates to the identification of shortcomings in the scope and standardization of CanL surveys, and how these can inform guidelines for future collection of prospective data.

Our best model for western Europe, the fourcountry model, predicted the seroprevalence of CanL based on 7 variables, namely country, altitude, minimum night-time land surface temperature (LSTNmn), amplitude of the tri-annual cycle of the night-time land surface temperature (LSTNa3), amplitude of the annual cycle of the Enhanced vegetation index (EVIa1), phase of the annual cycle of the Enhanced vegetation index (EVIp1) and phase of the tri-annual cycle of the Enhanced vegetation index (EVIp3). The association with CanL seroprevalence was inversely U-shaped for 4 of the predictors (altitude, LSTNmn, LSTNa3, and to a lesser degree EVIp1), and this can be explained by the ecological requirements of the nocturnal sandfly vectors being met in rural settings on the lower and middle slopes of hills and mountains (often 100-800 $\mathrm{m}$ a.s.1.) during the dry Mediterranean summer (Ready, 2008; Gálvez et al. 2010a; Mahamdallie et al. 2011). The more linear associations with EVIa1 and EVIp3 were negative and positive, respectively, and these findings should be explored by sandfly ecologists. There is no published risk map for CanL in the western Mediterranean to compare with our own. However, our risk map does appear to be inconsistent with the incidence map of
Trotz-Williams and Trees (2003), principally by not predicting more high risk areas near the coastal plains of Spain and Portugal, and also by predicting too many high risk areas in southwest, northwest and central France. Chamaillé et al. (2010) used the machine-learning Maxent method to produce an ecological niche model and a risk map for CanL in southern France, and this was based on presence data from our database, including many records from veterinary clinics. Our risk maps differ from theirs by identifying more low-high risk areas in central France and the inland parts of southwest France, as well as by identifying fewer medium-high risk areas in the more coastal parts of southwest France, where the Atlantic influences the climate.

One of the predictors of CanL in our four-country model was country. It is often the case that models differ between nearby countries, and this is true for anthroponotic visceral leishmanisis caused by Leishmania donovani in East Africa (Ready, 2008). Model variation between European countries might be explained by geographical differences in climate and seasonality associated with the bioclimatic and ecological requirements of the sandfly species serving as regional vectors. These considerations prompted us to construct a separate model and risk map for France, where both vectors ( $P$. perniciosus and $P$. ariasi) have overlapping altitudinal ranges throughout much of the Mediterranean region (Ready, 2008; Chamaillé et al. 2010; Hartemink et al. 2011), unlike in Italy where 1 of the 2 main vectors ( $P$. perfiliewi, not $P$. perniciosus) occurs only in the centre-south of the country (Maroli et al. 2008). Our best model for France alone, the France-only model, predicted the seroprevalence of CanL based on 6 variables, namely altitude, LSTNmn, amplitude of the bi-annual cycle of the night-time land surface temperature (LSTNa2), LSTNa3, amplitude of the annual cycle of middle infrared reflectance (MIRa1) and amplitude of the tri-annual cycle of middle infrared reflectance (MIRa3). LSTNa2 and MIRa3 were negatively associated with CanL seroprevalence, while the other 4 variables were positively associated with it. The France-only model differs from the four-country model mainly in the replacement of EVI, an index that estimates plant structural variation based on the reflectance of red and nearinfrared wavelengths, with MIR, an index that distinguishes better between active vegetation on the one hand and senescent vegetation, soils, rocks or anthropogenic surfaces on the other (Scharlemann et al. 2008).

The France-only model was found to be better than the four-country model for predicting the seroprevalence of CanL in France, probably because the latter was based mostly on surveys and dogs from Italy ( $40 \%$ and $84 \%$, respectively) rather than France (18\% and $7 \cdot 8 \%$, respectively). The four-country model was a poor global predictor of point values of 
CanL seroprevalence (correlation coefficient, $r=0 \cdot 341$ ), although the correlation was stronger for survey-rich Italy $(r=0.432)$ than for the other 3 countries $(r=0 \cdot 233-0 \cdot 262)$. In comparison, the France-only model gave a much better correlation between the predicted and observed CanL seroprevalences $(r=0.648)$. Based on AUC statistics, both models did provide fair-to-good discrimination (AUC >0.7) for the 2 extreme classes of CanL endemicity $(<5 \%,>20 \%$ seroprevalence) but not for the intermediate class (5-20\%). Many observed seroprevalences fell within this intermediate class (360 out of 947 overall, and 84 out of 169 in France), which is also true for much of the Mediterranean region (Dujardin et al. 2008), and so it is important to consider whether this lack of resolution results from the modelling approach or inadequate sampling.

The statistical methods commonly used to predict the occurrence or distribution of parasitic diseases in relation to environmental variables include discriminant (Rogers and Randolph, 2006) and Bayesian methods (Clements et al. 2006) as well as logistic regression, both binary (Brooker et al. 2001) and binomial (Rabe-Hesketh and Everitt, 2004). Binary logistic regression was used successfully for the spatial modelling of anthroponotic visceral leishmaniasis in East Africa (Thomson et al. 1999). Nonlinear discriminant analysis (NLDA) might provide better predictions than logistic regression (Rogers and Randolph, 2006), and it would be interesting to apply it to our datasets. However, we do not believe that NLDA would much improve the resolution of our predictions for those locations where CanL seroprevalence is in the intermediate range of $5-20 \%$. This conclusion stems from the limitations of our climate and RS datasets as well as of the sampling procedures used to obtain our survey data. In common with most spatial models, ours relied on using widely available sets of climate and RS data, which unfortunately cover different time periods and have varying spatial resolutions. None of the resolutions fully captured the variation in the microclimates inhabited by sandflies, which would hinder the inclusion of sandfly density as an explanatory variable in any integrated model (Hartemink et al. 2011). We could have modelled CanL seroprevalence over shorter time periods using matching climate surfaces from the EU ENSEMBLES project (http://www. ensembles-eu.org/), in order to improve the risk maps and investigate any effects of climate change. However, this requires the availability of CanL seroprevalence datasets with fewer limitations than those currently available, as now explained.

Our models' poor predictions of CanL seroprevalence in the intermediate range of $5-20 \%$ relate to shortcomings in the scope and standardization of CanL surveys. Firstly, there has been a bias towards known rural and peri-urban foci in Mediterranean bioclimate zones, and consequently zero seroprevalence was recorded in only $14.7 \%$ of the surveys (14-25\% in France, Portugal and Spain, but just $6 \%$ in Italy). We recommend that future surveys do not neglect environments at more extreme altitudes $(<100 \mathrm{~m},>1000 \mathrm{~m}$ a.s.1.) and northerly latitudes. Secondly, the most used serological test (IFAT) has not been standardized, e.g. the proportion of surveys using a cut-off of $<1 / 80$ was $35 \cdot 4 \%$ in Italy and $19 \cdot 2 \%$ in Portugal, but negligible in Spain $(2 \cdot 4 \%)$ and France $(0 \%)$. The use of low IFAT cut-offs in many surveys in Italy could possibly have produced for this country the highest median seroprevalence $(17 \cdot 7 \% ; 5 \cdot 9-8 \%$ elsewhere $)$ and the lowest frequency of zero seroprevalence $(6 \% ; 14-25 \%$ elsewhere). We strongly recommend that all published surveys include the proportion of dogs seropositive for each of the serial dilutions of the sera, not just for those making the cut-off, so that records from different periods can be better compared. Improvements in fluorescence microscopy have led to changes in the consensus cut-off, first $1 / 40$ in the $1970 \mathrm{~s}$, then $1 / 80$ in the $1990 \mathrm{~s}$, and sometimes $1 / 160$ recently. Thirdly, serology only detects a small fraction of resistant dogs and the susceptibles progressing to clinical disease after long incubation (Maia and Campino, 2008). Therefore, ideally, serology should be complemented by molecular screening in horizontal surveys (Lachaud et al. 2002; Quinnell and Courtenay, 2009). Lastly, the resolution of the sampling is inadequate, because a point location was very often an assembly point in a large village, to which dogs were brought for screening from a radius of $10 \mathrm{~km}$ or more (Rioux and Golvan, 1969; Maroli et al. 2008; Martín-Sánchez et al. 2009; Gálvez et al. 2010b). To improve the sampling resolution, we recommend including in the model 'dog factors', such as the age and lifestyle of individual dogs recorded as living most of the time in specific geo-referenced habitations. Concerning lifestyle, guard dogs and hunting dogs often have a higher prevalence of CanL than pet dogs that sleep indoors (Lanotte et al. 1978; Martín-Sánchez et al. 2009; Gálvez et al. 2010b). A potentially important explanatory variable missing from our modelling is dog density. Estimates have been used for ecological niche modelling (Chamaillé et al. 2010) and $\mathrm{R}_{0}$ modelling (Hartemink et al. 2011) of CanL or its vectors in southern France, but these estimates of dog density were based on human population densities that were low and varied little per $\mathrm{km}^{2}$ in the rural areas characteristic of CanL.

As a result of these shortcomings, our risk maps can not be expected to have much predictive power within many CanL foci. It might not always be cost effective to produce high resolution risk maps within CanL foci, for which it will be necessary to follow all our recommendations, but the consequences of not doing so ought to be assessed when agreeing guidelines for collecting prospective data. In 
contrast, it should be less challenging to improve spatial models so that different geographical regions can be compared. Then, our relatively straightforward method of producing risk maps would be more useful for predicting any northward emergence of CanL and helping to plan barrier methods of control, based currently on topical insecticides (deltamethrinimpregnated dog collars and pour-ons) not dog culling (Quinnell and Courtenay, 2009) and hopefully on vaccines (Ready, 2010). Already, we have demonstrated that country-level risk maps can distinguish well between areas with high (>20\%) and low $(<5 \%)$ CanL seroprevalences (only 3\% misclassified using the France-only model) and that seroprevalences are invariably low in areas of CanL emergence. For example, seroprevalence is usually $<5 \%$ throughout northern Italy above latitude $45 \cdot 5^{\circ}$ $\mathrm{N}$, where CanL has emerged within the last 20 years (Maroli et al. 2008). The improvement of spatial models for predicting CanL emergence in Europe depends on carrying out new standardized serological and molecular surveys in central and northern Europe as well as at extreme altitudes $(<100 \mathrm{~m}$, $>1000 \mathrm{~m}$ a.s.1.) in Mediterranean locations with comparable land covers. Without such sampling, predictions will not be possible for many regions of interest, such as much of France above $47 \cdot 5^{\circ} \mathrm{N}$, which is the approximate northern limit of our records and of the risk map produced by ecological niche modelling (Chamaillé et al. 2010). The complexities of predicting the effects of climate change (Kovats et al. 2001) lessen the likelihood of risk maps being used to predict the spread of CanL in response to global warming.

\section{ACKNOWLEDGEMENTS}

This work is dedicated to Professor Clive R. Davies, who initiated the research but passed away in March 2009. We thank the other members of the EDEN-Leishmaniasis subproject who helped to construct the database, namely Maria Antoniou and the team at the University of Heraklion (Greece), Robert Farkas and his team in the Faculty of Veterinary Science of Budapest (Hungary), and Yusuf Ozbel and his team in the University of Ege (Turkey). We are grateful for advice on statistical analyses from Francesco Checchi and Simon Brooker of the London School of Hygiene and Tropical Medicine, London, as well as Archie Clements and Ricardo Magalhães of the School of Population Health, University of Queensland, Australia.

\section{F I NANCIAL SUPPORT}

This work was funded by a grant (GOCE-2003-010284) of the European Union awarded to the EDEN (Emerging Diseases in a changing European eNvironment) project. The publication is catalogued by the EDEN Steering Committee as EDEN0262 (www.edenfp6project.net). Its contents are the sole responsibility of the authors and do not necessarily reflect the views of the European Commission. The funders had no role in study design, data collection and analysis, decision to publish, or preparation of the manuscript. The authors have declared that no competing interests exist.

\section{REFERENCES}

Brooker, S., Hay, S. I., Issae, W., Hall, A., Kihamia, C. M., Lwambo, N. J., Wint, W., Rogers, D. J. and Bundy, D. A. (2001). Predicting the distribution of urinary schistosomiasis in Tanzania using satellite sensor data. Tropical Medicine \& International Health 6, 998-1007. Brooker, S., Hay, S. I. and Bundy, D. A. (2002). Tools from ecology: useful for evaluating infection risk models? Trends in Parasitology 18, 70-74. Chamaillé, L., Tran, A., Meunier, A., Bourdoiseau, G., Ready, P. and Dedet, J.-P. (2010). Environmental risk mapping of canine leishmaniasis in France. Parasites \& Vectors 3, 31.

Clements, A.C., Moyeed, R. and Brooker, S. (2006). Bayesian geostatistical prediction of the intensity of infection with Schistosoma mansoni in East Africa. Parasitology 133, 711-719.

Dujardin, J. C., Campino, L., Cañavate, C., Dedet, J. P., Gradoni, L., Soteriadou, K., Mazeris, A., Ozbel, Y. and Boelaert, M. (2008). Spread of vector-borne diseases and neglect of Leishmaniasis, Europe. Emerging Infectious Diseases 14, 1013-1018.

Duprey, Z. H., Steurer, F. J., Rooney, J.A., Kirchhoff, L. V., Jackson, J. E., Rowton, E. D. and Schantz, P. M. (2006). Canine visceral leishmaniasis, United States and Canada, 2000-2003. Emerging Infectious Diseases 12, 440-446.

Gálvez, R., Descalzo, M. A., Miró, G., Jiménez, M. I., Martín, O., Dos Santos-Brandao, F., Guerrero, I., Cubero, E. and Molina, R. (2010a). Seasonal trends and spatial relations between environmental/meteorological factors and leishmaniosis sand fly vector abundances in Central Spain. Acta Tropica 115, 95-102.

Gálvez, R., Miró, G., Descalzo, M. A., Nieto, J., Dado, D., Martín, O., Cubero, E. and Molina, R. (2010b). Emerging trends in the seroprevalence of canine leishmaniasis in the Madrid region (central Spain). Veterinary Parasitology 169, 327-334.

Gething, P. W., Noor, A. M., Gikandi, P. W., Hay, S. I., Nixon, M. S., Snow, R. W. and Atkinson, P. M. (2008). Developing geostatistical spacetime models to predict outpatient treatment burdens from incomplete national data. Geographical Analysis 40, 167-188.

Hartemink, N., Vanwambeke, S. O., Heesterbeek, H., Rogers, D., Morley, D., Pesson, B., Davies, C., Mahamdallie, S. and Ready, P. (2011). Integrated mapping of establishment risk for emerging vector-borne infections: a case study of canine leishmaniasis in southwest France. PLoS ONE 6, e20817.

Hastie, T., Tibshirani, R. and Friedman, J. (2001). Model assessment and selection. In The Elements of Statistical Learning; Data Mining, Inference and Prediction, pp. 214-217. Springer, New York, USA.

Hay, S. I., Guerra, C. A., Gething, P.W., Patil, A. P., Tatem, A. J., Noor, A. M., Kabaria, C. W., Manh, B. H., Elvazar, I. R., Brooker, S., Smith, D. L., Moyeed, R. A. and Snow, R. W. (2009). A world malaria map: Plasmodium falciparum endemicity in 2007. PLoS Med 6, e1000048. Hosmer, D. W. and Lemshow, S. (2000). Applied Logistic Regression. John Wiley \& Sons, New York, USA

Kovats, R.S., Campbell-Lendrum, D. H., McMichael, A. J., Woodward, A. and Cox, J. S. (2001). Early effects of climate change: do they include changes in vector-borne disease? Philosophical Transactions of the Royal Society of London, B 356, 1057-1068.

Lachaud, L., Chabbert, E., Dubessay, P., Dereure, J., Lamothe, J., Dedet, J.-P. and Bastien, P. (2002). Value of two PCR methods for the diagnosis of canine visceral leishmaniasis and the detection of asymptomatic carriers. Parasitology 125, 197-207.

Lanotte, G., Rioux, J.-A., Croset, H. and Vollhardt, Y. (1978). Ecology of leishmaniasis in southern France. 9. Sampling methods in the study and analysis of canine enzootic leishmaniasis. Annales de Parasitologie Humaine et Comparée 53, 33-45.

Mahamdallie, S.S., Pesson, B. and Ready, P.D. (2011). Multiple genetic divergences and population expansions of a Mediterranean sandfly, Phlebotomus ariasi, in Europe during the Pleistocene glacial cycles. Heredity 106, 714-726.

Maia, C. and Campino, L. (2008). Methods for diagnosis of canine leishmaniasis and immune response to infection. Veterinary Parasitology 158, 274-287.

Maroli, M., Rossi, L., Baldelli, R., Capelli, G., Ferroglio, E., Genchi, C., Gramiccia, M., Mortarino, M., Pietrobelli, M. and Gradoni, L. (2008). The northward spread of leishmaniasis in Italy: evidence from retrospective and ongoing studies on the canine reservoir and phlebotomine vectors. Tropical Medicine \& International Health 13, 256-264. 
Martín-Sánchez, J., Morales-Yuste, M., Acedo-Sánchez, C., Barón, S., Diaz, V. and Morillas-Marquez, F. (2009). Canine leishmaniasis in southeastern Spain. Emerging Infectious Diseases 15, 795-798.

New, M., Lister, D., Hulme, M. and Makin, I. (2002). A high-resolution data set of surface climate over global land areas. Climate Research 21, 1-25. Pullan, R. L., Bethony, J.M., Geiger, S. M., Cundill, B., CorreaOliveira, R., Quinnell, R. J. and Brooker, S. (2008). Human helminth coinfection: analysis of spatial patterns and risk factors in a Brazilian community. PLoS Neglected Tropical Diseases 2, e352.

Quinnell, R. J. and Courtenay, O. (2009). Transmission, reservoir hosts and control of zoonotic visceral leishmaniasis. Parasitology 136, 1915-1934. Rabe-Hesketh, S. and Everitt, B. (2004). A Handbook of Statistical Analyses Using Stata, $3^{\text {rd }}$ Edn. Chapman \& Hall/CRC, Boca Raton, FL, USA

Ready, P.D. (2008). Leishmaniasis emergence and climate change. In Climate Change: Impact on the Epidemiology and Control of Animal Diseases (ed. De la Roque, S.) Revue Scientifique et Technique, Office International des Épizooties/ Scientific and Technical Review, World Organization for Animal Health 27, 399-412.

Ready, P. D. (2010). Leishmaniasis emergence in Europe. Eurosurveillance 15, e19505.

Ribeiro, P. J. Jr, and Diggle, P. J. (2001). geoR: a package for geostatistical analysis. R-NEWS $1,15-18$.

Rioux, J. A. and Golvan, Y. J. (1969). Épideémiologie des leishmanioses dans le sud de la France. Institut National de la Santé et de la Recherche Médicale, Paris.

Rogers, W. H. (1993). Regression standard errors in clustered samples. Stata Technical Bulletin 13, 19-23. (Available: http://www.stata.com/ products/stb/journals/stb13.pdf. Accessed 11 October 2009)
Rogers, D. J., Hay, S. I., and Packer, M. J. (1996). Predicting the distribution of tsetse flies in West Africa using temporal Fourier processed meteorological satellite data. Annals of Tropical Medicine and Parasitology 90, 225-241.

Rogers, D. J. and Randolph, S.E. (2006). Climate change and vectorborne diseases. In Global Mapping of Infectious Diseases: Methods, Examples and Emerging Applications (ed. Hay, S. I., Graham, A. J. and Rogers, D.J.), pp. 345-381. Academic Press, London, UK.

Romero, G. A. and Boelaert, M. (2010). Control of visceral leishmaniasis in Latin America - a systematic review. PLoS Neglected Tropical Diseases 4, e584.

Royston, P., Ambler, G. and Sauerbrei, W. (1999). The use of fractional polynomials to model continuous risk variables in epidemiology. International Fournal of Epidemiology 28, 964-974.

Scharlemann, J.P., Benz, D., Hay, S. I., Purse, B. V., Tatem, A. J., Wint, G. R. and Rogers, D. J. (2008). Global data for ecology and epidemiology: a novel algorithm for temporal Fourier processing MODIS data. PLoS One 3, e1408.

Thomson, M. C., Elnaiem, D. A., Ashford, R. W. and Connor, S. J. (1999). Towards a kala azar risk map for Sudan: mapping the potential distribution of Phlebotomus orientalis using digital data of environmental variables. Tropical Medicine \& International Health 4, 105-113.

Tibshirani, R., Hastie, T., Narasimhan, B. and Chu, G. (2002). Diagnosis of multiple cancer types by shrunken centroids of gene expression. Proceedings of the National Academy of Science,s USA 99 6567-6572.

Trotz-Williams, L. A. and Trees, A. J. (2003). Systematic review of the distribution of the major vector-borne parasitic infections in dogs and cats in Europe. Veterinary Record 152, 97-105. 